\title{
Opposing functions of the plant TOPLESS gene family during SNC1-mediated autoimmunity
}

\author{
Christopher M. Garner ${ }^{1,2,3 \times a}$, Benjamin J. Spears $\oplus^{1,3 \pm b}$, Jianbin Su ${ }^{1,3}$, Leland J. Cseke ${ }^{1,3}$, \\ Samantha N. Smith ${ }^{1,3}$, Conner J. Rogan $\circledast^{2,38 \mathrm{c}}$, Walter Gassmann $\circledast^{1,3 *}$ \\ 1 Division of Plant Sciences, University of Missouri, Columbia, Missouri, United States of America, 2 Division \\ of Biological Sciences, University of Missouri, Columbia, Missouri, United States of America, 3 Christopher S. \\ Bond Life Sciences Center and Interdisciplinary Plant Group, University of Missouri, Columbia, Missouri, \\ United States of America \\ a Current address: Thermo Fisher Scientific, Carlsbad, California, United States of America \\ ab Current address: Butler University, Indianapolis, Indiana, United States of America \\ ac Current address: Oregon State University, Corvallis, Oregon, United States of America \\ * gassmannw@missouri.edu
}

\section{f openaccess}

Citation: Garner CM, Spears BJ, Su J, Cseke LJ, Smith SN, Rogan CJ, et al. (2021) Opposing functions of the plant TOPLESS gene family during SNC1-mediated autoimmunity. PLoS Genet 17(2): e1009026. https://doi.org/10.1371/journal. pgen. 1009026

Editor: Gitta Coaker, University of California Davis, UNITED STATES

Received: August 2, 2020

Accepted: February 5, 2021

Published: February 23, 2021

Peer Review History: PLOS recognizes the benefits of transparency in the peer review process; therefore, we enable the publication of all of the content of peer review and author responses alongside final, published articles. The editorial history of this article is available here: https://doi.org/10.1371/journal.pgen.1009026

Copyright: @ 2021 Garner et al. This is an open access article distributed under the terms of the Creative Commons Attribution License, which permits unrestricted use, distribution, and reproduction in any medium, provided the original author and source are credited.

Data Availability Statement: All relevant data are within the manuscript and its Supporting Information files.

\section{Abstract}

Regulation of the plant immune system is important for controlling the specificity and amplitude of responses to pathogens and in preventing growth-inhibiting autoimmunity that leads to reductions in plant fitness. In previous work, we reported that SRFR1, a negative regulator of effector-triggered immunity, interacts with SNC1 and EDS1. When SRFR1 is nonfunctional in the Arabidopsis accession Col-0, SNC1 levels increase, causing a cascade of events that lead to autoimmunity phenotypes. Previous work showed that some members of the transcriptional co-repressor family TOPLESS interact with SNC1 to repress negative regulators of immunity. Therefore, to explore potential connections between SRFR1 and TOPLESS family members, we took a genetic approach that examined the effect of each TOPLESS member in the srfr1 mutant background. The data indicated that an additive genetic interaction exists between SRFR1 and two members of the TOPLESS family, TPR2 and TPR3, as demonstrated by increased stunting and elevated $P R 2$ expression in srfr1 tpr2 and srfr1 tpr2 tpr3 mutants. Furthermore, the tpr2 mutation intensifies autoimmunity in the auto-active snc1-1 mutant, indicating a novel role of these TOPLESS family members in negatively regulating $S N C 1$-dependent phenotypes. This negative regulation can also be reversed by overexpressing TPR2 in the sifr1 tpr2 background. Similar to TPR1 that positively regulates snc1-1 phenotypes by interacting with SNC1, we show here that TPR2 directly binds the N-terminal domain of SNC1. In addition, TPR2 interacts with TPR1 in vivo, suggesting that the opposite functions of TPR2 and TPR1 are based on titration of SNC1TPR1 complexes by TPR2 or altered functions of a SNC1-TPR1-TPR2 complex. Thus, this work uncovers diverse functions of individual members of the TOPLESS family in Arabidopsis and provides evidence for the additive effect of transcriptional and post-transcriptional regulation of SNC1. 
Funding: This research was funded by a University of Missouri Life Sciences Graduate Fellowship (CMG), a Division of Plant Sciences Daniel F. Millikan Fellowship (BJS), the Life Sciences Undergraduate Research Opportunity Fellows Program (CJR), and National Science Foundation (nsf.gov) grant IOS-1456181 (WG). The funders had no role in study design, data collection and analysis, decision to publish, or preparation of the manuscript.

Competing interests: The authors have declared that no competing interests exist.

\section{Author summary}

The immune system is a double-edged sword that affords organisms with protection against infectious diseases but can also lead to negative effects if not properly controlled. Plants only possess an innate antimicrobial immune system that relies on rapid upregulation of defenses once immune receptors detect the presence of microbes. Plant immune receptors known as resistance proteins play a key role in rapidly triggering defenses if pathogens breach other defenses. A common model of unregulated immunity in the reference Arabidopsis variety Columbia-0 involves a resistance gene called SNC1. When the $\mathrm{SNC} 1$ protein accumulates to unnaturally high levels or possesses auto-activating mutations, the visible manifestations of immune overactivity include stunted growth and low biomass and seedset. Consequently, expression of this gene and accumulation of the encoded protein are tightly regulated on multiple levels. Despite careful study the mechanisms of $S N C 1$ gene regulation are not fully understood. Here we present data on members of the well-known TOPLESS family of transcriptional repressors. While previously characterized members were shown to function in indirect activation of defenses, TPR2 and TPR 3 are shown here to function in preventing high defense activity. This study therefore contributes to the understanding of complex regulatory processes in plant immunity.

\section{Introduction}

Plants defend against infection by having a multilayered immune system, one branch of which recognizes molecular signatures of microbes through pattern recognition receptors at the cell surface. At the same time, plants monitor potential intracellular targets of pathogen attack $[1,2]$. At the heart of this intracellular plant surveillance system are the resistance genes of the nucleotide binding site-leucine-rich repeat (NLR) class [3]. Resistance proteins recognize, directly or indirectly, the actions of pathogen-secreted effector proteins which seek to interfere with plant immune responses or normal plant physiology. Upon sensing the activity of effectors, resistance proteins elicit a rapid and robust defense response, called effector-triggered immunity (ETI). In the case of the biotrophic defense response, this includes accelerated production of high levels of the plant hormone salicylic acid (SA) and the induction of PATHOGENESIS RELATED (PR) genes [1].

Because of cross-talk between plant hormone pathways, activation of the defense response is accompanied by repression of pathways that promote growth [4-7]. Therefore, induction of the plant immune system must be kept under tight control to avoid fitness penalties incurred during the absence of pathogen infection [8], as illustrated by autoimmune mutants of Arabidopsis that display the negative effects of an unregulated immune response. More than thirty different mutants have been identified that cause an autoimmune response exhibited by dwarfism, high levels of salicylic acid, constitutive defense gene expression, and subsequent increased resistance to pathogens [9]. Genetic analysis of these mutants has provided a wealth of information regarding the identity of positive and negative regulators of the immune response, and they illustrate the many levels of regulation that take place within the plant immune system.

SUPRESSOR of rps4-RLD1 (SRFR1) is a negative regulator of ETI mediated by several NLR proteins with a Toll/interleukin-1 receptor domain at their N-termini (TNLs), including RPS4/RRS1 and SNC1 [10-12]. It was discovered in a genetic screen for mutants that were resistant to Pseudomonas syringae pv. tomato strain DC3000 expressing the bacterial effector 
AvrRps4 in the Arabidopsis accession RLD, which is normally susceptible because of natural inactivating polymorphisms in the RPS4 resistance gene [10]. Mutants of $s r f r 1$ in the Col-0 background constitutively activate SNC1 expression, causing an autoimmune phenotype characterized by high levels of salicylic acid, constitutive expression of $P R$ genes, and severe stunting $[12,13]$. This autoimmune phenotype is absent in the RLD background due to an absence of a full-length SNC1 allele [12]. SRFR1 interacts with the TNLs RPS4, RPS6, and SNC1 as well as the central ETI regulator EDS1 in a complex disrupted by AvrRps4 [2,14]. Furthermore, srfrl eds1 mutants lose increased resistance phenotypes [14]. These results place SRFR1 as a key regulator of effector-triggered immunity conferred by the TNL class of resistance genes.

In addition to interactions within an ETI protein complex, homology to transcriptional regulators and interaction with transcription factors suggest SRFR1 could also be part of a transcriptional repressor complex [11]. SRFR1 interacts with members of the TEOSINTE BRANCHED1-CYCLOIDEA-PROLIFERATING CELL FACTOR (TCP) transcription factor family in the nucleus. Specifically, SRFR1 interacts strongly with TCP8, TCP14, and TCP15, and a triple tcp $8 t c p 14 t c p 15$ mutant is compromised in effector-triggered immunity [15]. This interaction between SRFR1 and positive ETI regulators suggests a model wherein SRFR1 is restricting TCP access to promoters of defense-related genes, or recruiting other proteins that function as repressors of transcription at these promoters.

The five member Arabidopsis TOPLESS gene family (TPL, TOPLESS RELATED1, TPR2, TPR3, and TPR4) encodes members of the larger GRO/TUP1 family of corepressors that are proposed to interact with DNA-binding proteins in the promoter regions of regulated genes to repress transcription [16]. Analysis of TPL/TPR family interactions with transcription factors indicates that they have been coopted multiple times to regulate gene expression in diverse processes, including control of flowering time, hormone signaling, and stress responses [17]. Structural studies also provide evidence that TPL tetramerizes as part of its interactions with protein partners, suggesting the possibility of heterotetramers within the TOPLESS family [18].

Furthermore, TPR1 was shown to interact with SNC1, and together the complex, with an as yet unknown DNA-binding transcription factor, represses transcription of genes that function as negative regulators of defense responses such as DEFENSE NO DEATH 1 (DND1) and $D N D 2$, which encode cyclic nucleotide-gated ion channels [19,20]. Therefore, similar to the interactions of SRFR1 with the TNL-mediated ETI machinery and transcription factors, TOPLESS family members display multiple mechanisms in their functions as co-repressors.

Whether SRFR1 is acting as part of a complex with the ETI machinery or functions as a transcriptional co-repressor, which molecular pathways regulate the autoimmunity phenotype of srfrl mutants remains a pressing question. Both models presented us with the possibility that SRFR1 may also be interacting, at least genetically, with members of the TOPLESS family. Thus, we hypothesized that loss-of-function mutations in the TOPLESS gene family in the srfrl-4 background would display similar phenotypes to the $t p l / t p r 1$ mutants in the snc1-1 auto-active mutant background, reducing the SNC1-mediated autoimmune response. Here, we report the unexpected result that mutations in TPR 2 and TPR 3 have the opposite effect from those in $T P R 1$, increasing the $S N C 1$ autoimmune response in the $s r f r 1-4$ mutant background. This presents a novel function for TPR 2 and TPR 3 in either repressing positive regulators of the immune response or interfering with the SNC1-TPR1-mediated repression of negative regulators.

\section{Results}

\section{Mutations in TPR2 exacerbate the srfr 1-4 autoimmune phenotype}

To investigate possible genetic interactions between SRFR1 and members of the TOPLESS family, srfr1-4 was crossed with T-DNA mutants in TPL, TPR1, TPR2, TPR3, and TPR4. 
Homozygous $s r f r 1-4 \mathrm{tpl} / \mathrm{tpr}$ double mutants were compared to $s r f r 1-4$ to determine if stunting, a measure of constitutively activated defenses, was affected. To quantify these differences in stunting we also measured shoot weights from each genotype after 4 weeks of growth. The results showed that srfr1-4 tpl-8 and srfr1-4 tpr2-2 were significantly different from srfrl-4 in terms of size and overall shoot mass, in opposite directions (Fig 1). No difference in shoot
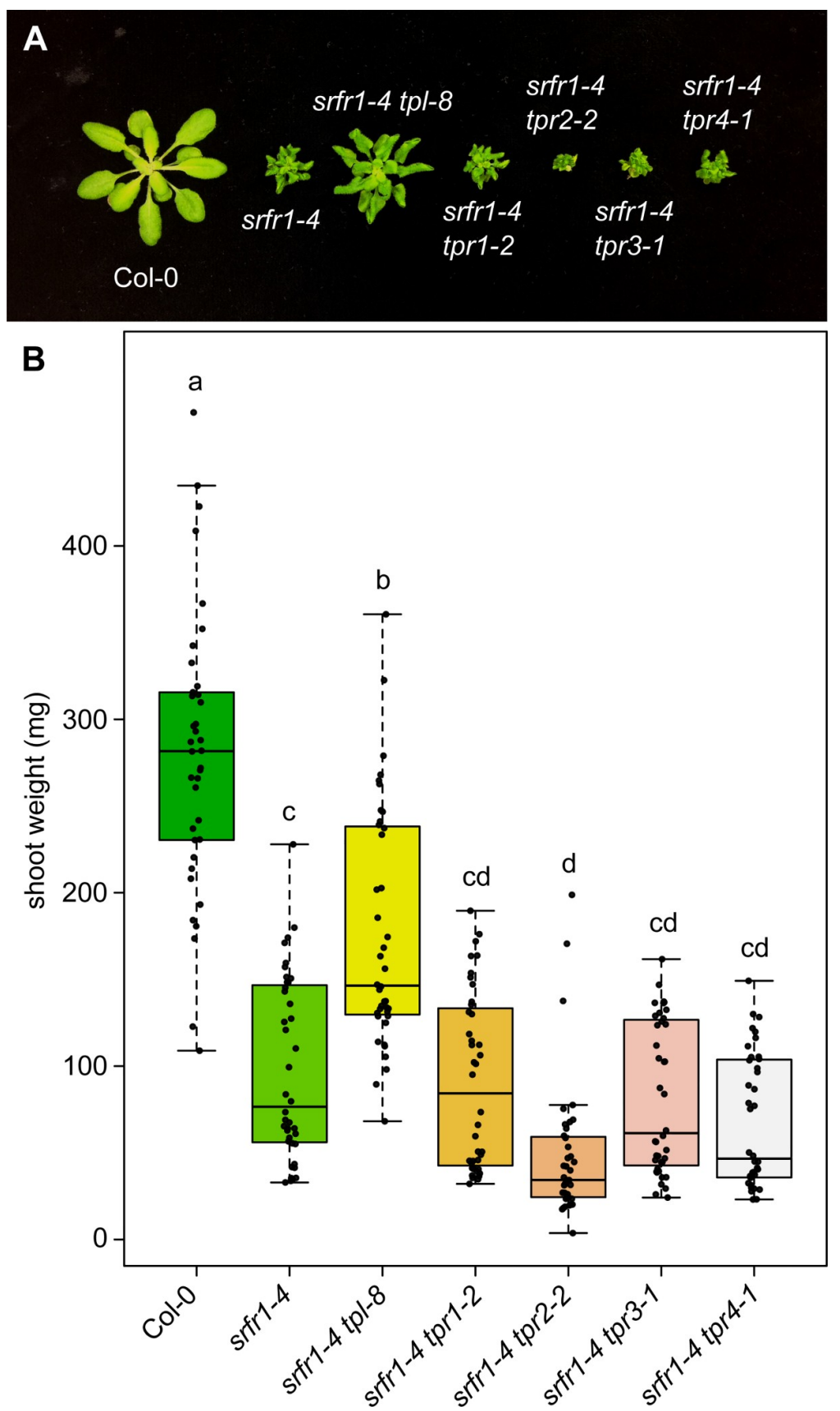

Fig 1. Loss of function of TPR2 increases stunting in srfr1. (A) Morphological phenotype of $s r f r 1-4$ and $s r f r 1-4 t p l /$ $t p r$ double mutants at four weeks post sowing. (B) Shoot weight from plants grown under short day conditions at $21^{\circ} \mathrm{C}$ for four weeks. Dots represent individual data points taken over two separate experiments. Whiskers on boxplots are drawn to the farthest data point within $1.5^{*} \mathrm{IQR}$ of first and third quartiles. Letters denote significant differences as determined by Student's t-test $(P<0.01)$ using the Bonferroni-Holm method to correct for multiple comparisons. 
mass was observed in the single tpl/tpr mutants compared to Col-0 (S1 Fig). PR2 is well established as an overall marker of immune system activation, and we found that the degree of stunting in this panel of auto-immune mutants correlated with their level of PR2 expression (S2 Fig).

Stunting in srfr1-4 is due to the activation of the TNL gene SNC1 [13,19]. Given that it was shown that mutation of $t p l$ lessens the effect of stunting in autoactive snc1-1 mutants [19], and the dependency of stunting in srfrl-4 on activation of $S N C 1$, we concluded that the effect we were seeing in srfrl-4 tpl-8 mutants was a recapitulation of previous findings and chose not to investigate this mutant further. We did not see a similar phenotype in srfr1-4 tpr1-2. The T-DNA insertion in the tpr1-2 allele occurred in an intron in the 5' untranslated region, and the absence of a phenotype indicates that this allele is not a complete knockout. In contrast, the increased stunting of srfr1-4 tpr2-2 represents a novel genetic interaction, and as such we switched our focus to concentrate on the SRFR1-TPR2 interaction. Stunting was alleviated in the srfr $1-4$ single and the $s r f r 1-4 \mathrm{tpl} / \mathrm{tpr}$ double mutants when plants were grown at higher temperature, consistent with growth phenotypes of other mutants with activated SNC1 [12]. This indicates that the increased stunting observed with srfr1-4 tpr2-2 is also dependent on SNC1 (S3 Fig).

To verify that the increased autoimmunity phenotype was indeed caused by the insertion at the TPR2 locus and not some other tightly linked mutation, we obtained a second allele of TPR2, tpr2-1, and crossed this allele to srfr1-4. For both tpr2 alleles we did not detect TPR2 mRNA (S4 Fig). As with srfr1-4 tpr2-2, we saw increased stunting in the srfr1-4 tpr2-1 double mutant relative to srfrl-4 (Fig 2A). To quantify these differences in stunting we measured shoot weights from each genotype after 4 weeks of growth. The results showed that srfrl-4 tpr2-1 and srfr1-4 tpr2-2 were significantly different from srfr1-4 in terms of overall shoot mass (Fig 2B), but that neither TPR2 single mutant was significantly different from Col-0.

\section{$T P R 2$ and $T P R 3$ are partially redundant or function additively in repressing autoimmunity in $s r f r 1-4$}

Previous research has demonstrated functional redundancy amongst TOPLESS family members, and that higher order $t p l / t p r$ knockouts produce stronger phenotypes than single $t p l / t p r$ mutants [21-23]. Based on the close evolutionary relatedness of TPR2 and TPR3 (S5 Fig) and previous reports that indicated TPL, TPR1, and TPR4 are repressors of negative regulators of immunity [19], we chose to investigate if mutations in TPR3 would impact the srfr1-4 tpr2-2 phenotype. To obtain a srfr1-4 tpr2-2 tpr3-1 triple mutant, srfr1-4 tpr2-2 was crossed with srfr1-4 tpr3-1. Analysis of shoot mass showed that the srfr1-4 tpr2-2 tpr3-1 triple mutant is significantly smaller than both srf1-4 and srfr1-4 tpr2-2 (Fig 3A and 3B).

As TOPLESS family members have been shown to be repressors of transcription we decided to examine the mRNA levels of SNC1 in the srfr1-4 tpr2-2 and srfr1-4 tpr2-2 tpr3-1 mutants to see if they were affected relative to $s r f r 1-4$. We also examined $P R 2$ expression as a marker for overall immune activation and used qPCR rather than protein blotting to quantify subtle differences in mRNA levels for the remainder of this study. As illustrated in Fig 3C and 3D, PR2 and SNC1 mRNA levels were significantly increased in srfr1-4 tpr2-2 and srfr1-4 tpr2-2 tpr3-1 relative to srfr1-4; however, no significant change in PR2 or SNC1 expression was observed in the tpr2-2 or tpr3-1 single mutants.

Given the partial redundancy or additive function observed between TPR 2 and TPR 3 in the srfr 1-4 background and the lack of any observable phenotype in the single mutants, we crossed tpr2-2 to tpr3-1 to create a tpr2-2 tpr3-1 double mutant. No stunting or other morphological phenotypes were observed in tpr2-2 tpr3-1 (Fig 4A). We also found no significant difference 


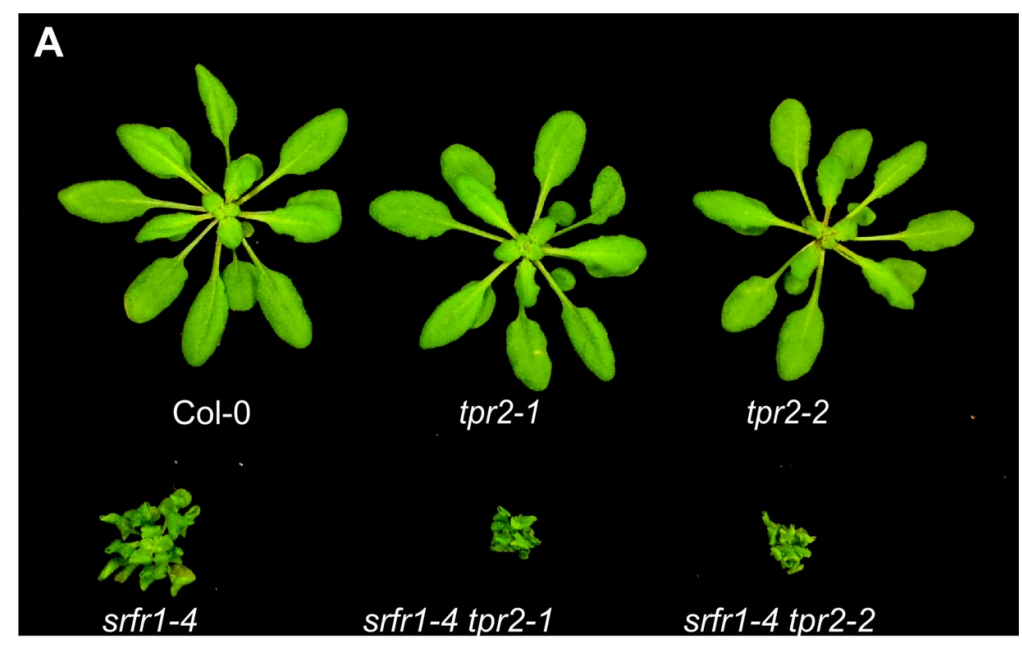

B

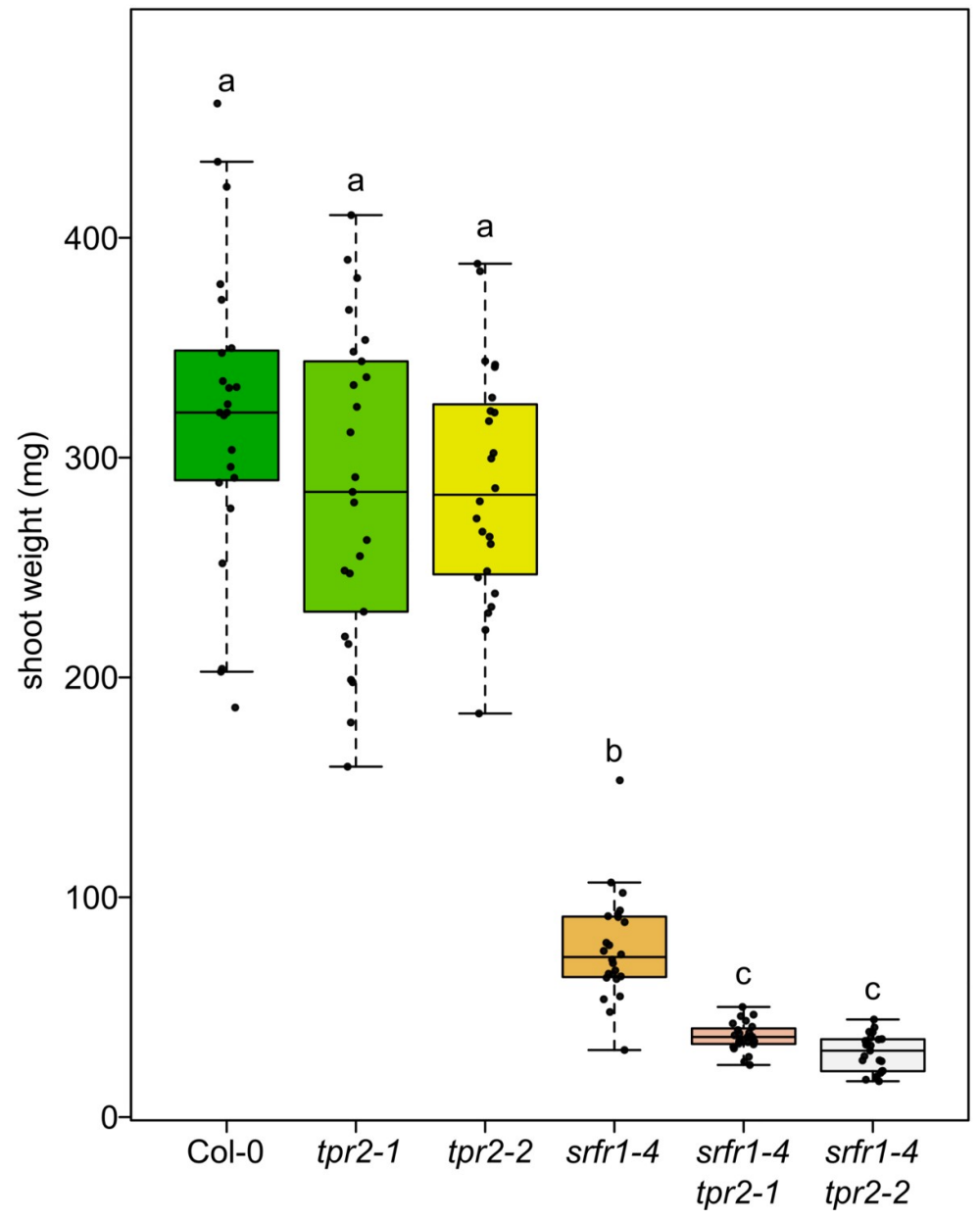

Fig 2. Multiple alleles of TPR2 increase stunting in srfr1. (A) Morphological phenotypes of tpr2-1, tpr2-2, srfr1-4, srfr1-4 tpr2-1, and srfr1-4 tpr2-2 at four weeks post sowing. (B) Shoot weight from plants grown under short day conditions at $21^{\circ} \mathrm{C}$ for four weeks. Dots represent individual data points. Whiskers on boxplots are drawn to the farthest data point within $1.5^{*}$ IQR of first and third quartiles. Letters denote significant differences as determined by Student's t-test $(P<0.05)$ using the Bonferroni-Holm method to correct for multiple comparisons.

https://doi.org/10.1371/journal.pgen.1009026.g002 

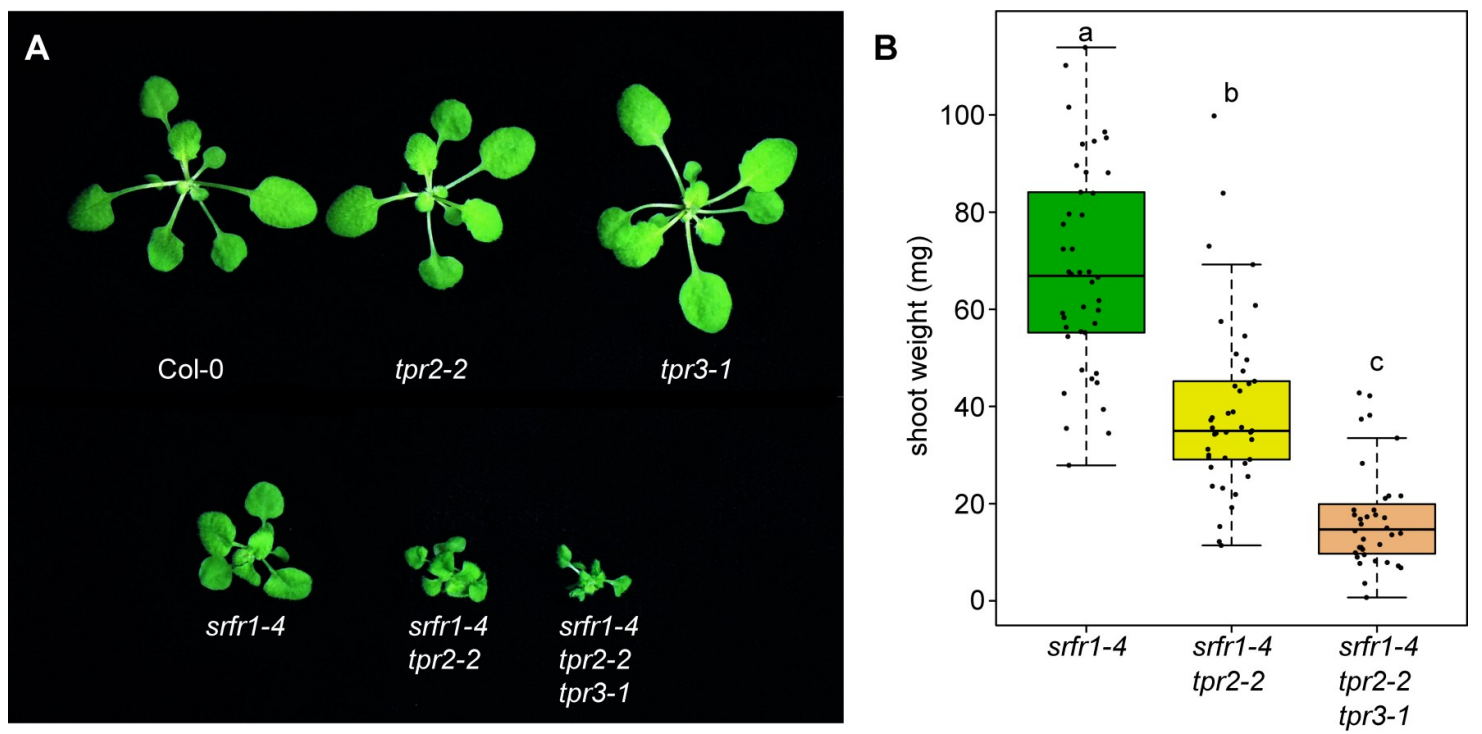

C

PR2

D

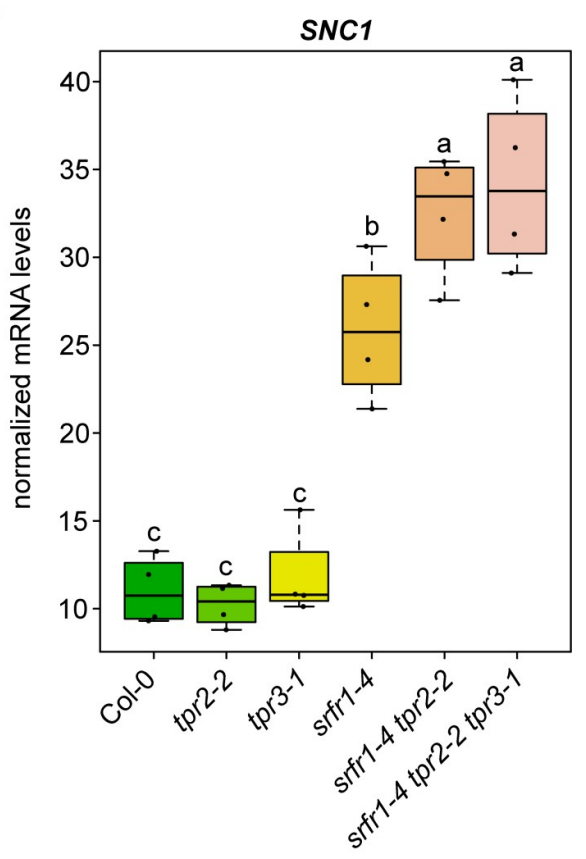

Fig 3. Simultaneous loss of TPR2 and TPR3 increases stunting and expression of $P R 2$ and $S N C 1$ in srfr1. (A) Morphological phenotype of srfr1-4, srfr1-4 tpr2-2, and srfr1-4 tpr2-2 tpr3-1 at 20 days after sowing. Plants were grown under short day conditions at $21^{\circ} \mathrm{C}$. (B) Shoot weight from plants grown under short day conditions at $21^{\circ} \mathrm{C}$ for four weeks. Dots represent individual data points taken over two separate experiments. Whiskers on boxplots are drawn to the farthest data point within $1.5^{*}$ IQR of first and third quartiles. Letters denote significant differences as determined by Student's t-test $(P<0.001)$ using the Bonferroni-Holm method to correct for multiple comparisons. (C\&D) Expression as measured by quantitative RT-PCR of PR2 and SNC1 in single, double, and triple mutants. Dots represent individual data points taken over two separate experiments. Genes of interest were normalized against SAND (At2g28390). Whiskers on boxplots are drawn to the farthest data point within $1.5^{*} \mathrm{IQR}$ of first and third quartiles. Letters denote significant differences as determined by Student's t-test $(P<0.05)$ using the Bonferroni-Holm method to correct for multiple comparisons.

https://doi.org/10.1371/journal.pgen.1009026.g003

between Col-0 and tpr2-2 tpr3-1 with regards to PR2 expression; however, we did see a small but significant increase in $S N C 1$ expression in tpr2-2 tpr3-1 when compared to Col-0 (Fig 4B and 4 C). Consistent with the molecular data we did not observe a difference in resistance to DC3000 in tpr2-2, tpr3-1, or tpr2-2 tpr3-1 compared to Col-0 (S6 Fig). 

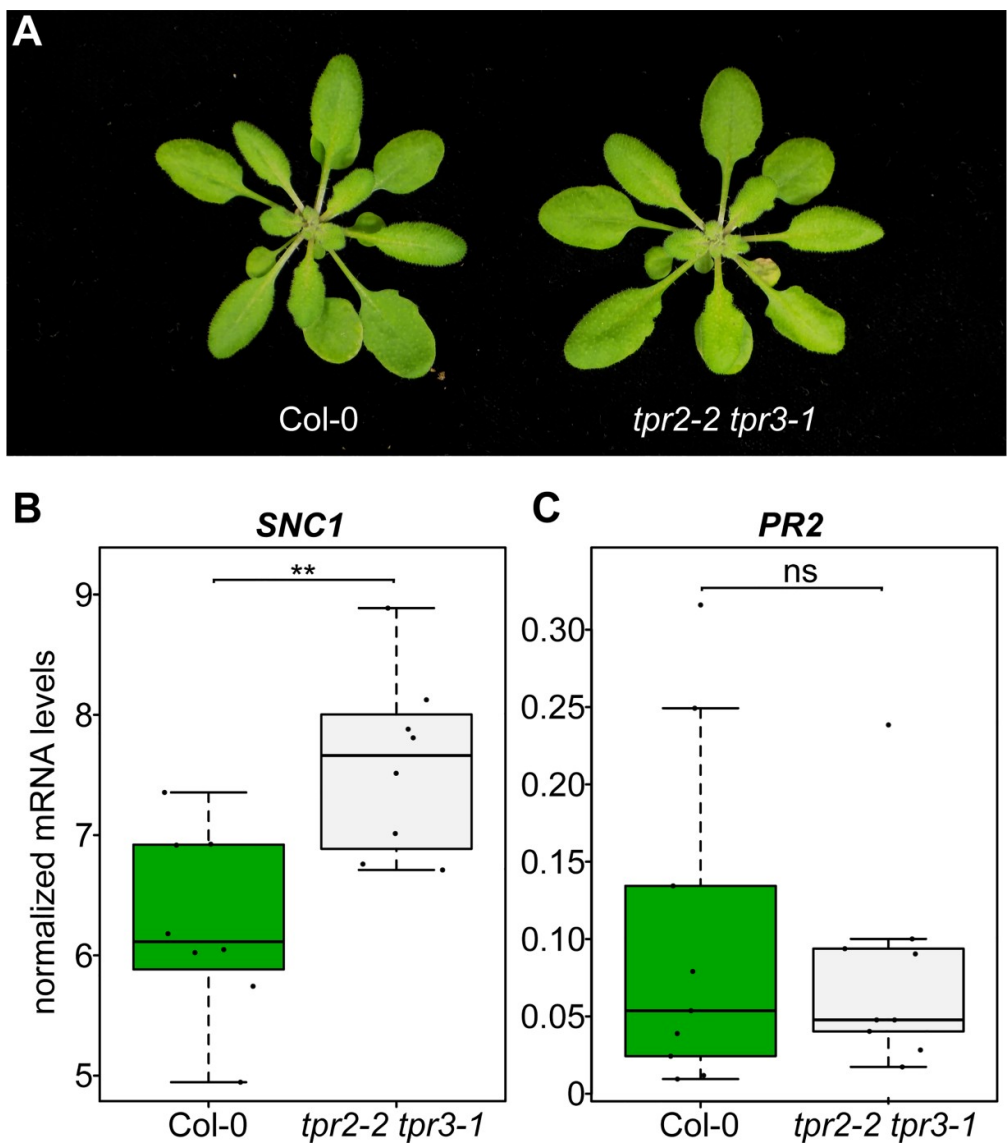

Fig 4. SNC1 expression is increased in tpr2 tpr3. (A) Morphological phenotype of tpr2-2 tpr3-1. Plants were grown for four weeks under short day conditions at $21^{\circ} \mathrm{C}$. (B\&C) Expression as measured by quantitative RT-PCR of PR2 and $S N C 1$. Dots represent individual data points taken over two separate experiments. Genes of interest were normalized against SAND (At2g28390). Whiskers on boxplots are drawn to the farthest data point within $1.5^{*}$ IQR of first and third quartiles. Asterisks denote significant differences as determined by Student's t-test $(P<0.005)$ using the Bonferroni-Holm method to correct for multiple comparisons.

https://doi.org/10.1371/journal.pgen.1009026.g004

\section{Overexpression of $T P R 2$ in the $s r f r 1-4$ background represses autoimmunity}

We next asked if overexpressing TPR2 would have the opposite effect and suppress autoimmunity in the srfr1-4 tpr2-2 background. To test this hypothesis we cloned the TPR2 coding sequence as a translational fusion with a C-terminal 10xMyc tag behind the constitutively active cauliflower mosaic virus $35 \mathrm{~S}$ promoter. Using the 35S:TPR2-myc construct, several stable lines were created in the srfr1-4 tpr2-2 genetic background. Two independent homozygous TPR2-myc srfr1-4 tpr2-2 lines in the T3 generation were planted alongside Col-0, srfr1-4, and srfr1-4 tpr2-2 to compare the degree of stunting. At four weeks after planting, the TPR2-myc srfr1-4 tpr2-2 plants were less stunted than both srfr1-4 tpr2-2 and srfr1-4 (Fig 5A).

Quantification of $S N C 1$ showed that not only was transcript level reduced below srfri-4 tpr2-2 levels, but was also less than $S N C 1$ levels in srfr1-4 (Fig 5B), correlating with plant size (Fig 5A). The TNL gene RPP4 is located within the SNC1 locus and has been shown to be coregulated with $S N C 1$ both at the level of transcription and after transcription by RNA silencing [24]. We have also previously shown that RPP4 is upregulated in srfr1-4 [12]. To determine if $T P R 2$ also affects $R P P 4$ expression in the srfr $1-4$ background, we quantified $R P P 4$ mRNA in srfr1-4 tpr2-2 and in TPR2-myc srfr1-4 tpr2-2. We saw a slight non-significant increase in 

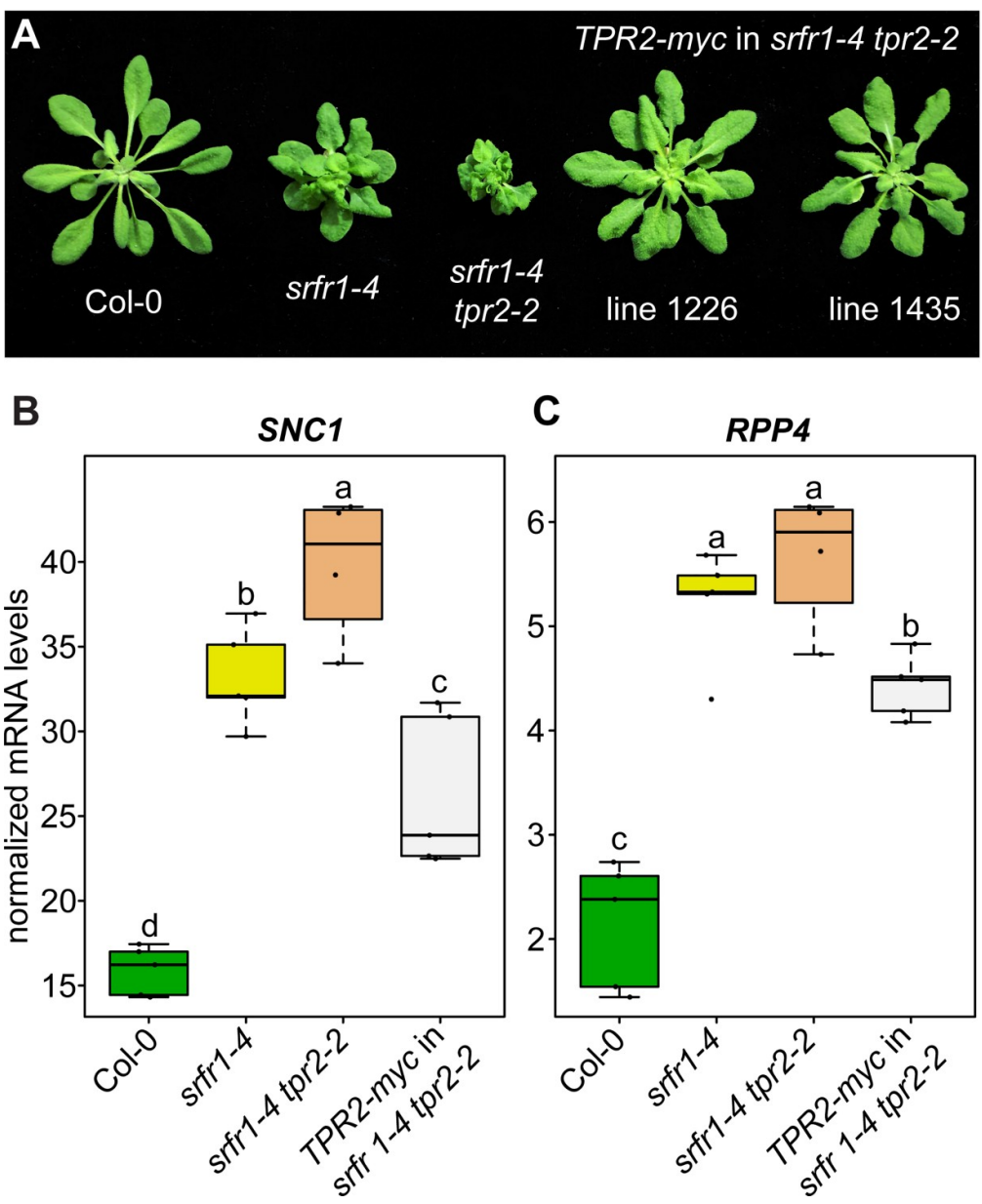

Fig 5. Overexpression of TPR2 reduces stunting and $S N C 1$ expression in srfr1 tpr2. (A) Morphological phenotype of TPR2-myc srfr1-4 tpr2-2 compared to srfr1-4 and srfr1-4 tpr2-2. Plants were grown under short day conditions at $21^{\circ} \mathrm{C}$ for four weeks. (B\&C) Expression as measured by quantitative RT-PCR of SNC1 and RPP4. Dots represent individual data points taken over two separate experiments. Genes of interest were normalized against SAND (At2g28390). Whiskers on boxplots are drawn to the farthest data point within $1.5 *$ IQR of first and third quartiles. Letters denote significant differences as determined by Student's t-test $(P<0.05)$ using the Bonferroni-Holm method to correct for multiple comparisons.

https://doi.org/10.1371/journal.pgen.1009026.g005

$R P P 4$ expression in srfr1-4 tpr2-2 relative to $s r f r 1-4$, while $R P P 4$ mRNA was reduced in TPR2-myc srfr1-4 tpr2-2 below levels in srfr1-4 (Fig 5C).

\section{Increased autoimmunity in srfr1-4 tpr2-2 is partially dependent upon SNC1}

Previous work has shown that stunting in srfrl-4 is dependent on SNC1, and that a srfrl-4 snc1-11 double mutant is morphologically normal but still expresses higher than normal levels of several defense-related genes [12]. To see if the enhanced autoimmunity that results from mutating TPR2 in the srfr $1-4$ background is dependent on $S N C 1$, we created a quadruple mutant by crossing the $S N C 1$ knockout allele, snc1-11, to srfr1-4 tpr2-2 tpr3-1. As was previously observed for srfr1-4 snc1-11, we saw no stunting or morphological abnormalities in the srfr1-4 snc1-11 tpr2-2 tpr3-1 quadruple mutant (Fig 6A). SRFR1 regulation of RPP4 is SNC1 independent as RPP4 is upregulated equally in both srfrl-4 and srfr1-4 snc1-11 relative to wild type levels in Col-0 [12]. Interestingly, RPP4 expression was significantly decreased both in srfr1-4 snc1-11 tpr2-2 tpr3-1 compared to srfr1-4 snc1-11 and in snc1-11 tpr2-2 tpr3-1 


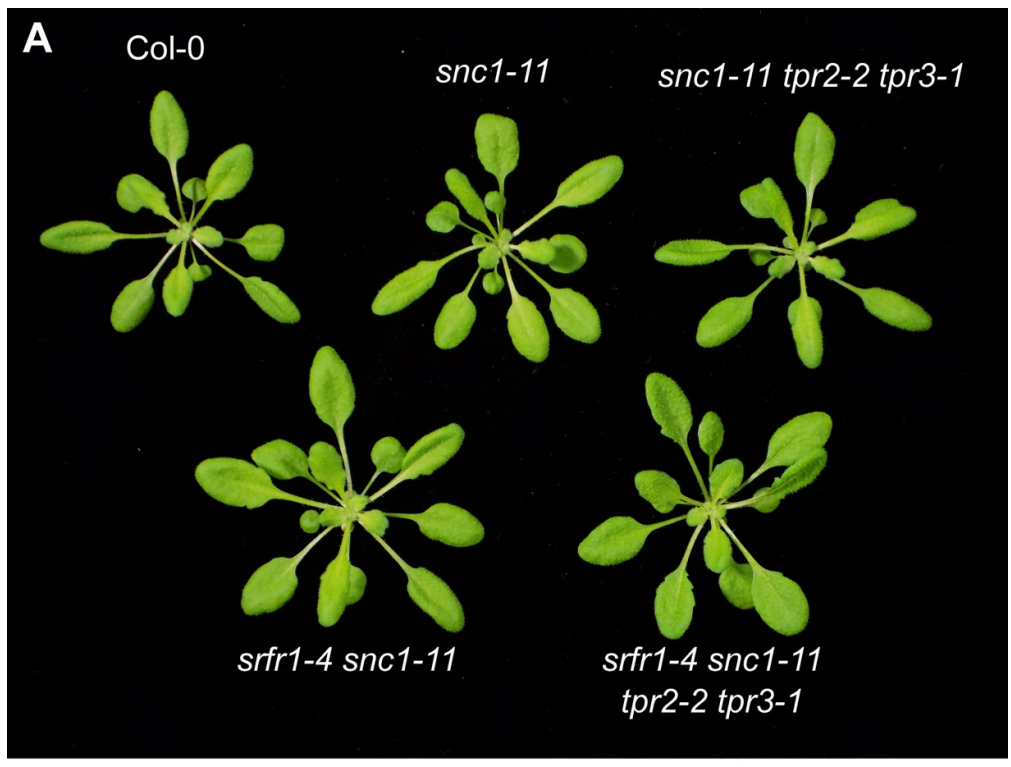

B

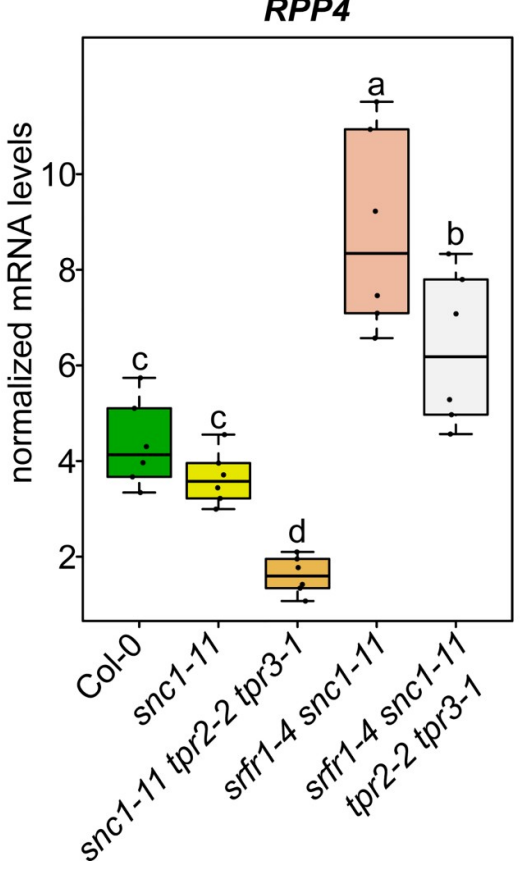

C

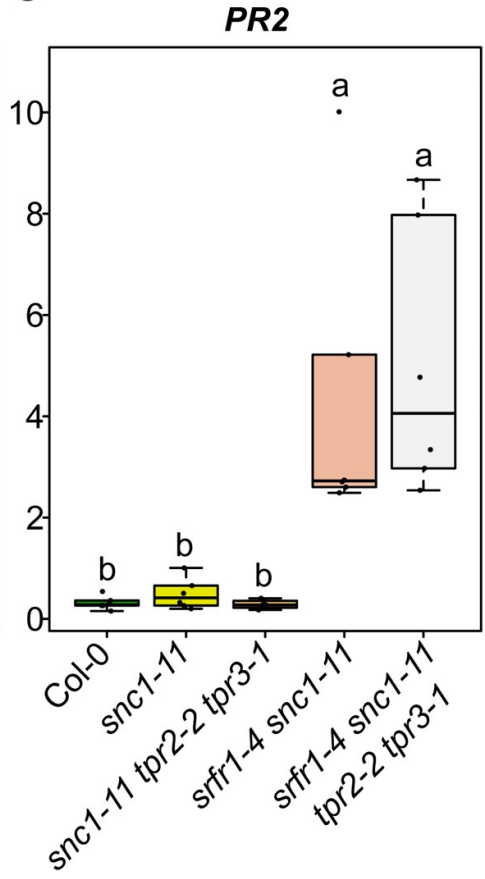

Fig 6. tpr 2 tpr3 mutants have lower expression of RPP4 in snc1 knockouts. (A) Morphological phenotype of plants harboring the snc1-11 mutation crossed into srfrl and tpr2 tpr3 mutants. Plants were grown under short day conditions at $21^{\circ} \mathrm{C}$ for four weeks. (B\&C) Expression as measured by quantitative RT-PCR of RPP4 and PR2. Dots represent individual data points taken over two separate experiments. Genes of interest were normalized against $S A N D$ (At2g28390). Whiskers on boxplots are drawn to the farthest data point within $1.5^{*}$ IQR of first and third quartiles. Letters denote significant differences as determined by Student's t-test $(P<0.05)$ using the Bonferroni-Holm method to correct for multiple comparisons.

\section{https://doi.org/10.1371/journal.pgen.1009026.g006}

compared to snc1-11 (Fig 6B), whereas RPP4 mRNA levels in the srfr1-4 tpr2-2 mutant were slightly higher than in srfrl-4 (Fig 5C), indicating that these higher RPP4 mRNA levels are at least partially dependent upon $S N C 1$. Consistent with our previous study, we saw an increase in PR2 levels in the srfr1-4 snc1-11 double mutant compared to Col-0 and snc1-11. PR2 levels 

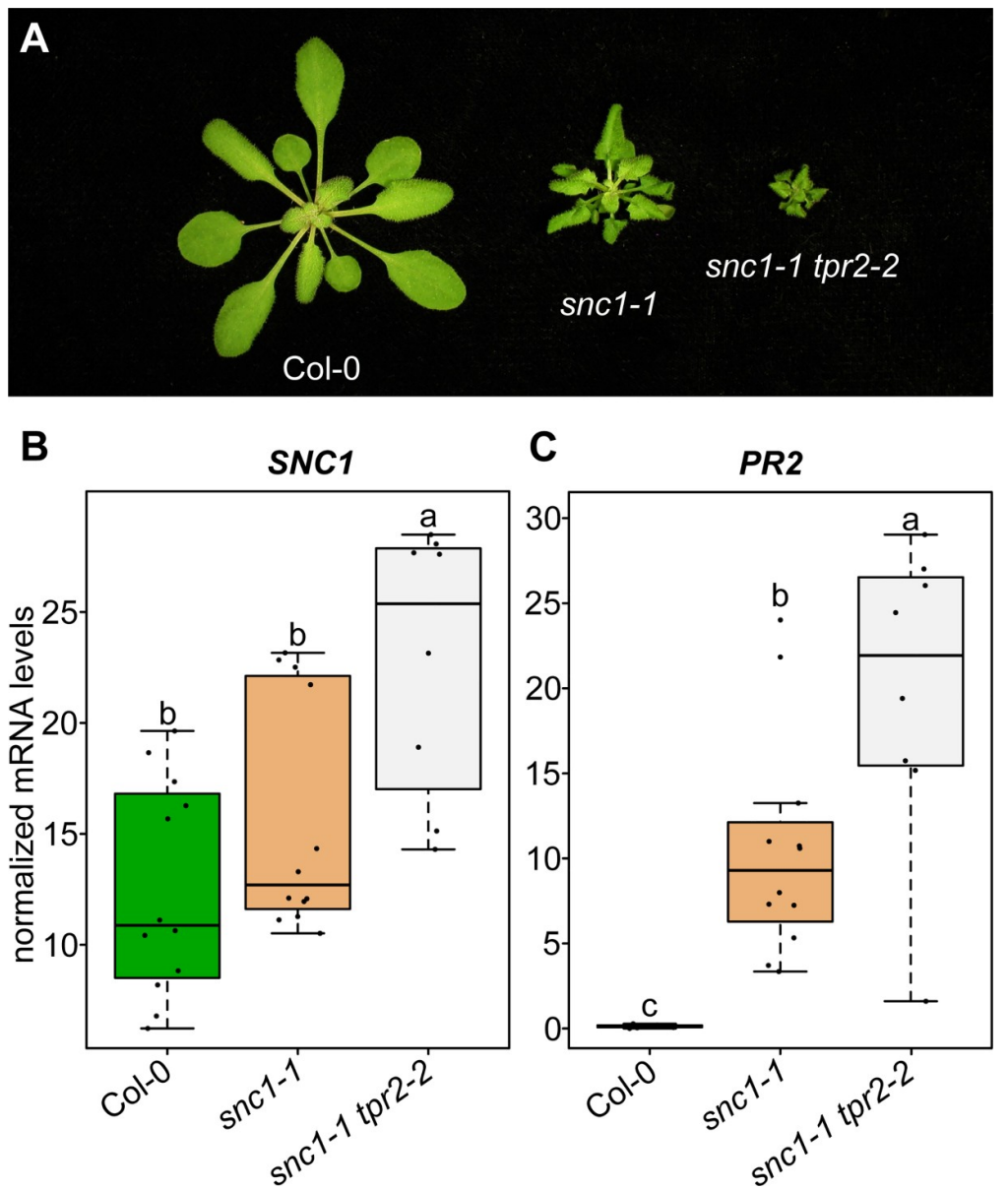

Fig 7. Mutations in TPR2 increase stunting and SNC1 expression in snc1-1 mutants. (A) Morphological phenotypes of snc1-1 and snc1-1 tpr2-2. Plants were grown under short day conditions at $21^{\circ} \mathrm{C}$ for four weeks. (B\&C) Expression as measured by quantitative RT-PCR of $S N C 1$ and $P R 2$. Dots represent individual data points taken over two separate experiments. Genes of interest were normalized against SAND (At2g28390). Whiskers on boxplots are drawn to the farthest data point within $1.5^{*} \mathrm{IQR}$ of first and third quartiles. Letters denote significant differences as determined by Student's t-test $(P<0.01)$ using the Bonferroni-Holm method to correct for multiple comparisons.

\section{https://doi.org/10.1371/journal.pgen.1009026.g007}

in srfr1-4 snc1-11 tpr2-2 tpr3-1 were comparable to those in srfr1-4 snc1-11 (Fig 6C), and in planta bacterial growth assays showed comparable levels of increased resistance (S7 Fig).

To further investigate the relationship between TPR2 and SNC1 activity, we crossed tpr2-2 to snc1-1, an auto-active allele of $S N C 1$ that induces a constitutive defense response and associated stunting [25]. The F2 from this cross produced approximately $1 / 16^{\text {th }}$ plants which genotyped as homozygous snc1-1 tpr2-2 that were extremely stunted and produced very little seed. When compared to $s n c 1-1, \operatorname{snc1-1}$ tpr2-2 was significantly more stunted, and had significantly higher levels of $S N C 1$ and $P R 2$ mRNA (Fig 7). These results are consistent with the conclusion that the autoimmune phenotypes modulated by mutations in SRFR1 and TPR2 are tightly associated with SNC1.

\section{SRFR1 acts upstream of SNC1 transcription}

Transcription of $S N C 1$ is subject to feedback regulation through the production of salicylic acid. Upon activation of SNC1, SA accumulates in the plant and increased levels of SA cause even more transcription of SNC1 [26]. Our data show that $t p r 2-2$ increases SNC1 mRNA levels 
in the srfr1-4 and snc1-1 backgrounds, but because of the complex feedback regulation of SNC1 transcription it is unclear whether SRFR1 and TPR2 are directly affecting transcription at the $\mathrm{SNC1}$ locus, or if they are repressing some component downstream of SNC1 activation. Signaling for all Arabidopsis TNL class resistance proteins identified to date is dependent upon EDS1 [27], and mutating EDS1 blocks the feedback regulation of SNC1, thereby making it possible to disambiguate events upstream of $S N C 1$ transcription from events downstream of SNC1 activation [28]. The eds1-2 allele is a knockout for EDS1 introgressed into Col-0 [29]. Previous work has shown that a srfr1-4 eds1-2 double mutant shows no signs of enhanced basal resistance and is morphologically indistinguishable from Col-0 [14].

To determine if the tpr2-2 mutation had any effect on transcription of $S N C 1$ in srfr1-4 eds12, we crossed eds1-2 to tpr2-2 and srfr1-4 tpr2-2 to srfr1-4 eds1-2 and obtained eds1-2 tpr2-2 and srfr1-4 eds1-2 tpr2-2 mutants. As seen previously with the srfr1-4 eds1-2 double mutant, the srfr1-4 eds1-2 tpr2-2 triple mutant was not morphologically different from Col-0 (Fig 8A). When we quantified the amount of $S N C 1$ transcript in these plants we found that $s r f r 1-4$ eds12 produced significantly more $S N C 1$ than Col-0, eds1-2, and eds1-2 tpr2-2 (Fig 8B). The srfr1-4 eds1-2 tpr2-2 triple mutant had a repeatable but non-significant decrease in $S N C 1$ relative to srfr1-4 eds1-2 (Fig 8B). These data suggest that SRFR1 also acts upstream of SNC1 transcription, while TPR2 acts downstream of $S N C 1$ transcription.

TPR1 was previously shown to directly interact with the TIR domain of SNC1 [19]. To determine if TPR2 interacts with SNC1, we performed an in vitro pull down assay between GST-tagged TPR2 and T7-tagged SNC1-TIR domain. Pull down of GST-TPR2 with GST beads co-precipitated T7-SNC1-TIR, whereas pull down of GST alone failed to co-precipitate T7-SNC1-TIR (Fig 8C), indicative of a direct protein-protein interaction between TPR2 and SNC1. TOPLESS family members are not known to heteromerize except for TPR1 and TPR4 [17]. We therefore thought it likely that the post-transcriptional activity of TPR2 consists of competing with TPR1 for binding of SNC1. To test this in vivo, we transiently expressed GFP-SNC1, myc-TPR1 and HA-TPR2 in Nicotiana benthamiana eds1-1 plants to minimize tissue disintegration by SNC1 activity [30]. Consistent with the in vitro data, both TPR1 and TPR2 were co-immunoprecipitated with SNC1 (Fig 9A). Interestingly, we discovered that TPR1 and TPR2 also interact with each other (Fig 9B). This suggests that the mechanism of TPR2 and TPR1 antagonism is based on titration of SNC1-TPR1 complexes by TPR2 or altered functions of a SNC1-TPR1-TPR2 complex.

\section{Discussion}

To determine whether members of the TPL transcriptional repressor gene family functionally interact with SRFR1 we chose a genetic approach. By creating double and higher order mutants between srfr1-4, members of the TOPLESS family, and other genes relevant to the srfrl-4 autoimmune phenotype, we were able to assess the impact these genes had on constitutive immunity. Our results indicate a genetic interaction between SRFR1 and TPR2 and its close homolog TPR3. Further data show a novel genetic interaction between SNC1 and TPR2. We found that stunting in $s r f r 1-4$ was affected by mutations in TPL and TPR2, but in opposite ways; srfrl-4 tpl-8 was less stunted, and srfr1-4 tpr2-2 was more stunted. To verify that these phenotypes were a consequence of altered immune system regulation, and not a developmental phenotype unrelated to defense, we measured the expression of $P R 2$ as a marker of the defense response [31,32]. Previous research has shown that $P R 1$ and $P R 2$ mRNA levels are elevated in srfrl-4 relative to wild type plants [12]. Here, we found that PR2 levels in srfr1-4 tpl-8 and srfr1-4 tpr2-2 are indeed consistent with differentially regulated immune system outputs in these double mutants. 


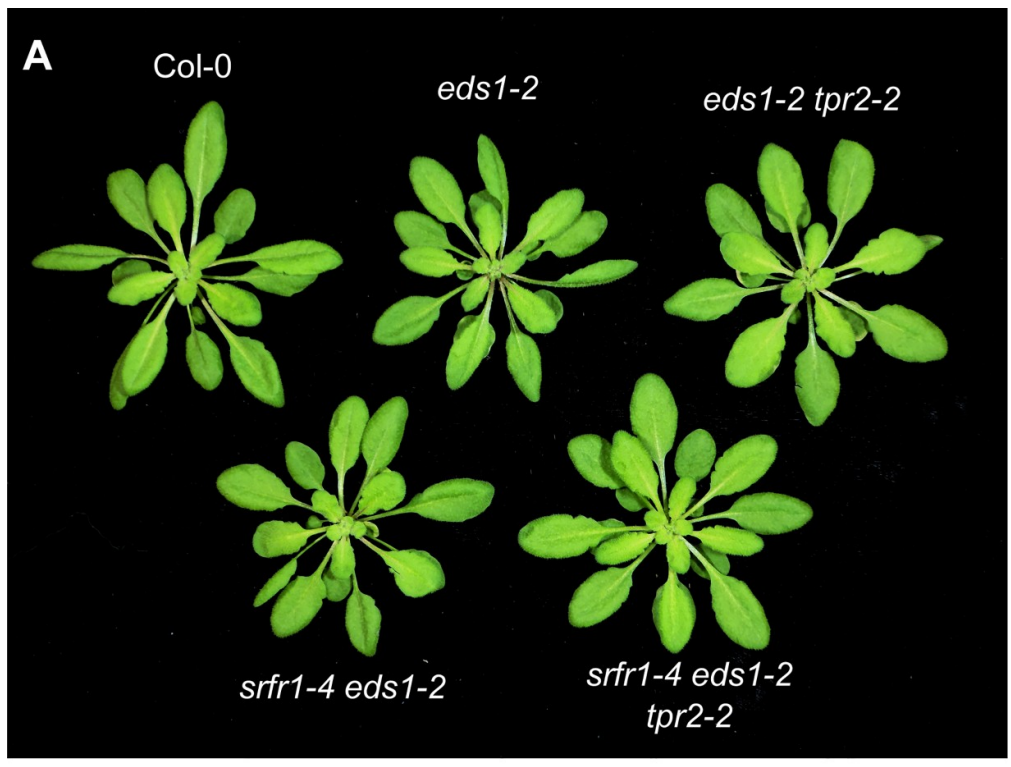

B

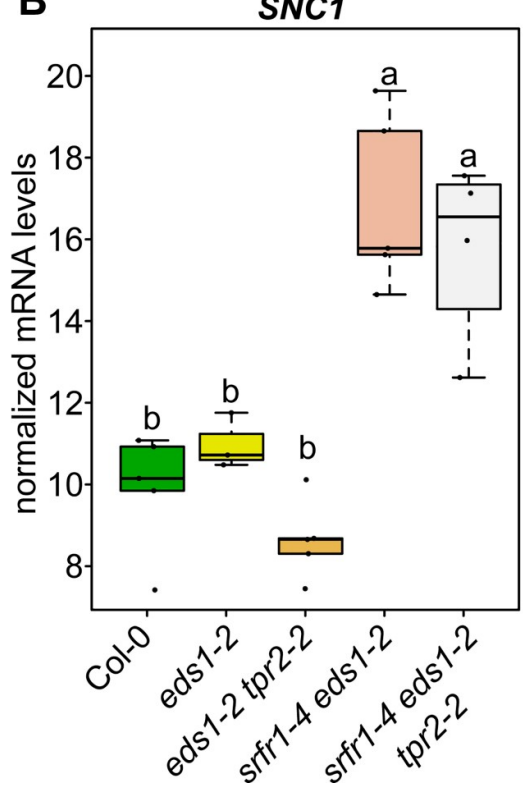

C
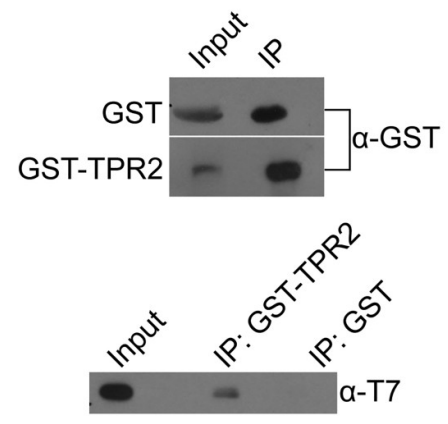

T7-SNC1-TIR

Fig 8. SRFR1 acts upstream of SNC1 transcription. (A) Morphological phenotypes of single, double, and triple mutants of $e d s 1-2, \operatorname{srfr} 1-4$, and tpr2-2. Plants were grown under short day conditions at $21^{\circ} \mathrm{C}$ for four weeks. (B) Expression as measured by quantitative RT-PCR of $S N C 1$. Dots represent individual data points taken over two separate experiments. Genes of interest were normalized against SAND (At2g28390). Whiskers on boxplots are drawn to the farthest data point within $1.5^{*} \mathrm{IQR}$ of first and third quartiles. Letters denote significant differences as determined by Student's t-test $(P<0.01)$ using the Bonferroni-Holm method to correct for multiple comparisons. (C) In vitro interaction of TPR2 and the TIR domain of SNC1 in E. coli. Proteins were pulled down and subjected to immunoblot analysis with either GST or T7 antibodies. This experiment was repeated once with similar results. https://doi.org/10.1371/journal.pgen.1009026.g008

\section{Contrasting roles of $T P R 1 / T P L$ and $T P R 2 / T P R 3$}

Stunting, but not all aspects of heightened basal resistance in srfrl-4 has been previously shown to be dependent upon the TNL gene SNC1 [12]. One mechanism by which SNC1 activates the immune system was demonstrated to be through a protein interaction with TPR1, the end result of this interaction being the repression of negative regulators of defense such as 
A

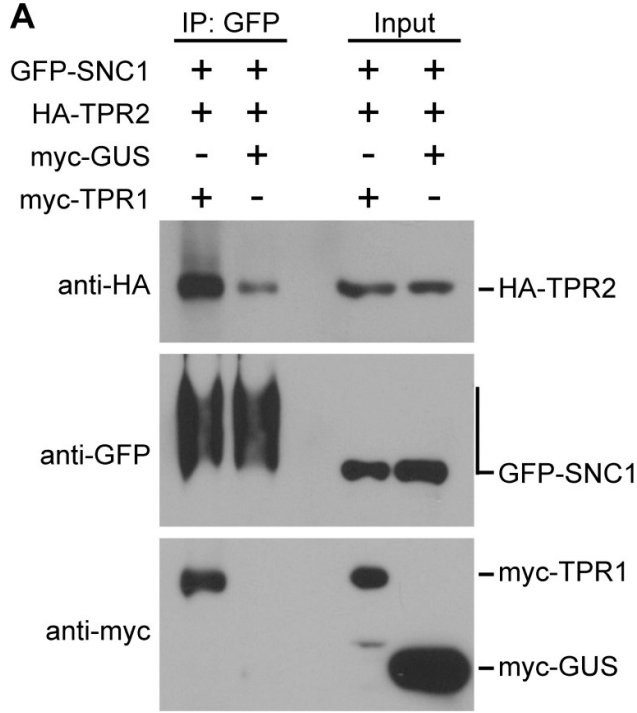

B

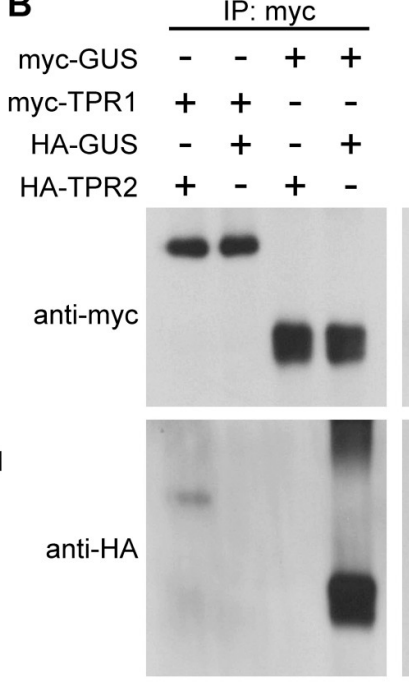

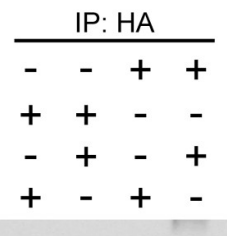

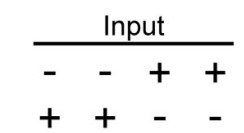

$++-$

$-+-+$

$+-+$
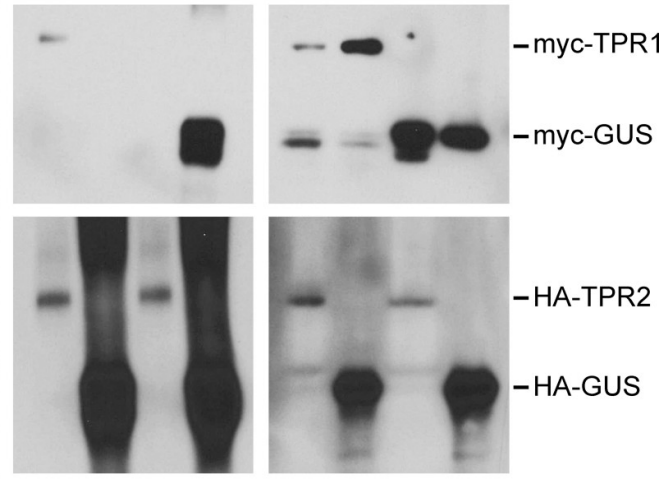

Fig 9. SNC1, TPR1 and TPR2 interact with each other in vivo. Tagged proteins were expressed in N. benthamiana eds1-1 plants. (A) Immunoprecipitation of GFP-SNC1 and detection of co-immunoprecipitated myc-TPR1, and of HA-TPR2 in the presence or absence of myc-TPR1, with myc-GUS as a control. (B) Reciprocal co-immunoprecipitation of myc-TPR1 and HA-TPR2, with correspondingly tagged GUS as negative (interaction with TPR) and positive (self-interaction) control. The expected protein band positions based on molecular size markers are indicated to the right of blots.

https://doi.org/10.1371/journal.pgen.1009026.g009

DND1 and DND2. SNC1 was also shown to interact genetically with $T P L$, which shares $92 \%$ identity with TPR1 at the amino acid level [19]. The attenuated autoimmunity we observed in srfrl-4 tpl-8 is in agreement with this model. We did not see a similar phenotype in srfrl-4 tpr1-2, most likely because the tpr1-2 allele is not a true knockout. We verified by sequencing out from the T-DNA that the location of the tpr1-2 insertion is within the first intron of TPR1, which is located in the 5 ' untranslated region. This insertion may not be sufficient to knock out transcription of functional TPR1 mRNA.

In contrast to $s r f r 1-4 t p l-8$, the $s r f r 1-4$ tpr2-2 phenotype is a novel case wherein a member of the TOPLESS family is implicated in repressing an immune response. Based on the strikingly different phenotypes of the double mutants we propose that TPR2 is repressing a set of genes disparate from that of TPR1 or is activating genes in the srfr1-4 background. We verified that the exacerbated autoimmune phenotype in srfr $1-4$ tpr2-2 was linked to TPR 2 by demonstrating that another allele of TPR2, tpr2-1, could produce the same phenotype in srfr1-4.

Previous research has shown varying degrees of redundancy amongst the different members of the TOPLESS family depending on the process under study. In embryogenesis and circadian clock regulation, knocking out all TPL/TPR genes is required to see a phenotype $[21,33]$, whereas, for partial alleviation of repression of brassinosteroid-sensitive genes via BZR1, the tpl tpr1 tpr4 triple mutant was sufficient [23]. Mutants of TPR2 and TPR3 were not included in this analysis and the partial nature of the tpl tprl tpr4 mutant phenotype was assumed to be based on the presence of functional TPR2 and TPR3 [23]. A certain degree of redundancy may also explain the relatively weak effects of the majority of $t p r$ single mutants on srfr1-4-mediated phenotypes. In addition, TPR3, the closest homolog of TPR2, has some functional redundancy with TPR2 in repressing autoimmunity in srfr1-4 in that the srfr $1-4$ tpr2-2 tpr3-1 triple mutant is significantly more stunted than srfr1-4 tpr2-2 and shows increased PR2 levels relative to srfr1-4 tpr2-2 and srfr1-4. It is a common observation that gene family members of transcriptional regulators display redundancy and that subsets of members effect opposite regulation, as in the cases of WRKY and TCP transcription factors in plant 
immunity [34]. However, the context-specific function of TPR2/TPR3 as either redundant with or opposite to TPL/TPR1/TPR4 depending on the regulatory pathway is surprising.

\section{Contributions to $S N C 1$ regulation by $S R F R 1$}

Although stunting in srfr1-4 is fully dependent upon SNC1, SRFR1 has a broader effect on immune function independent of SNC1. The TNL resistance genes RPS4, RPP4, and At4g16950 are all upregulated in $s r f r 1$ mutants independent of $S N C 1$, as well as several other genes related to immune function such as EDS1, PAD4, SID2, PR1, and PR2 [11,12]. SNC1 is located within the RPP5 disease resistance locus, a complex locus containing several paralogous resistance genes [35]. It has been previously shown that activation of SNC1 leads to increased transcription of other resistance genes at this locus, such as RPP4 and At4g16950 $[12,24,36]$. The mechanism by which RPP4 and At4g16950 are upregulated by activated SNC1 is unknown, although two possibilities were proposed in Yi and Richards. The first involves upregulation as a result of increased SA caused by SNC1 activation, citing previous work showing that application of SA is sufficient to cause a large increase in SNC1 transcript [26]. However, they also do not rule out the possibility that chromatin structure at the locus might be altered due to increased transcription of $S N C 1$, creating a permissive environment for transcription of neighboring paralogs [24].

Interestingly, RPP4 and At4g16950 are both upregulated in srfr1-4 snc1-11 [12], a genetic background without a functional copy of $S N C 1$, and as a consequence of this observation we hypothesized that the PR2 increase we observed in srfr1-4 snc1-11 tpr2-2 tpr3-1 could be due to a further increase in transcript of these other RPP5 locus resistance genes. Surprisingly, $R P P 4$ levels were significantly decreased by adding the tpr2 and tpr3 mutations to srfr $1-4$ snc111 , implying that the increased RPP4 in srfr1-4 tpr2-2 relative to $s r f r 1-4$ is fully dependent upon increased $S N C 1$. We therefore asked if TPR2 had a genetic interaction with $S N C 1$ by crossing tpr2-2 with snc1-1. The snc1-1 allele contains a point mutation in the linker region between the NBS and LRR domains that causes constitutive activation of the SNC1 protein and associated stunting caused by induction of the defense response without increasing the levels of snc1-1 mRNA $[25,37]$. In the snc1-1 tpr2-2 double mutant we saw significantly increased stunting, and snc1-1 and PR2 mRNA levels, suggesting a role for TPR2 in the downregulation of the SNC1-mediated constitutive defense response.

In order for resistance genes of the TNL class to function, the lipase like protein EDS1 must be present [38-40]. To elucidate the position of TPR2 in the SNC1-mediated constitutive defense response we took advantage of the srfr1-4 eds1-2 double mutant which blocks increased basal resistance in srfr1-4 [14] and consequently feedback upregulation of SNC1.

Other studies have used mutations in EDS1, and closely related protein interactor PAD4 which is also required for $S N C 1$ signaling, to block feedback upregulation of $S N C 1$ to determine if genes are acting upstream or downstream of SNC1 activation [25,26,28,41]. In the srfr1-4 eds12 tpr2-2 triple mutant we did not see a significant increase in SNC1 mRNA absent of SNC1 protein activation compared to $s r f r 1-4$ eds1-2. This result implies that TPR2 is acting downstream of SNC1 activation, whereas SRFR1 also impacts the level of SNC1 mRNA. This difference may be one component for the additive effect of mutations in SRFR1 and TPR2 on the level of constitutively activated defenses.

\section{Model for TPR2/TPR3 and SRFR1 functions in SNC1-mediated autoimmunity}

Based on these data we present the following model for TPR2 and SRFR1 function in autoimmunity caused by SNC1 activation (Fig 10). In the srfr1-4 background SNC1 mRNA is 


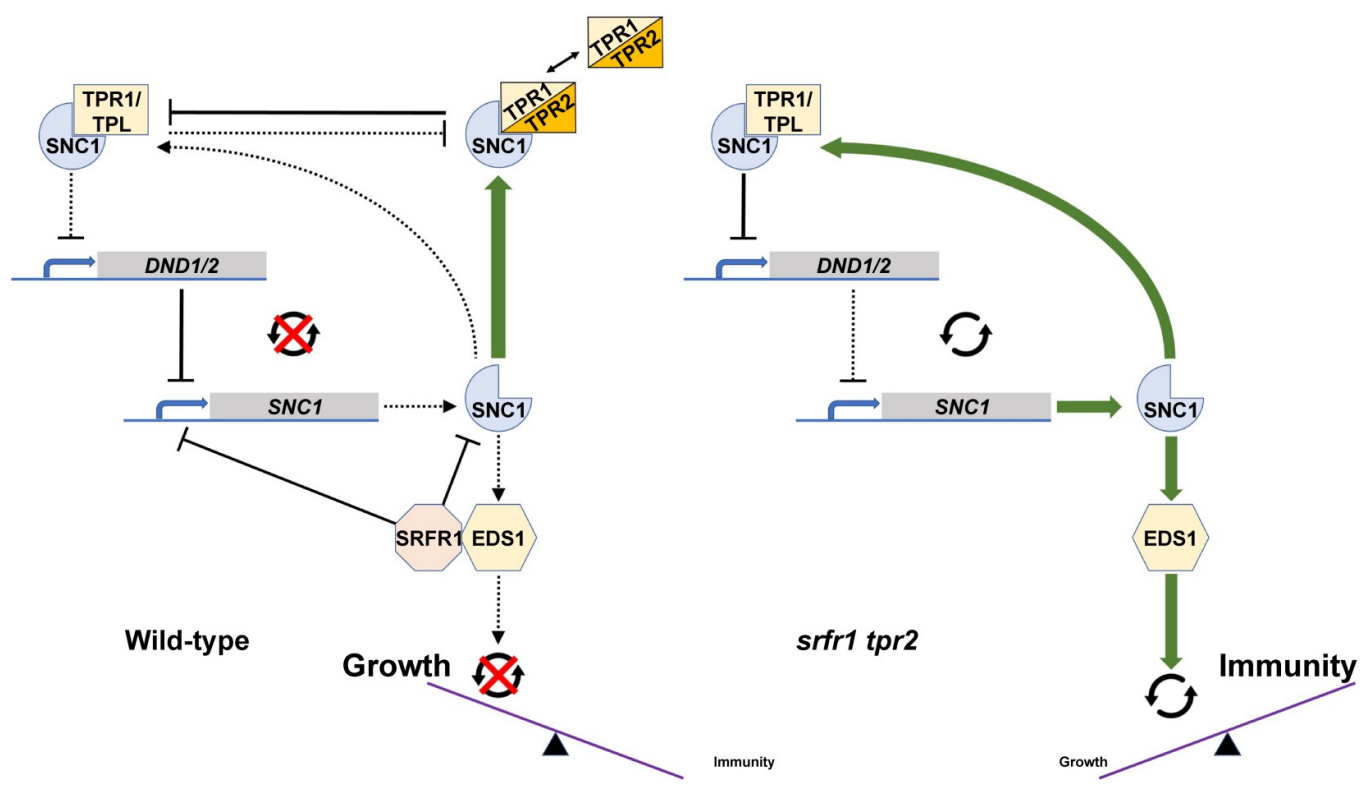

Fig 10. Model for TPR2 and SRFR1 functions in SNC1-mediated autoimmunity. (Left) In Col-0, low levels of SNC1 help to avoid fitness penalties. This may be accomplished both through direct inhibition by SRFR1 and through sequestration by TPR2 of TPR1 to reduce TPR1-SNC1 interactions that affect negative regulators of immunity such as DND1/DND2 and indirectly subsequent SNC1 expression, or through formation of a SNC1-TPR1-TPR2 complex with altered functions compared to an inhibitory SNC1-TPR1 complex. Here, the combined effects of SRFR1 and TPR2 hold SNC1 expression in check. (Right) In the srfr1-4 tpr2-2 double mutant, these molecular check points are released, allowing SNC1 expression to trigger an autoimmune response that results in excessive stunting.

https://doi.org/10.1371/journal.pgen.1009026.g010

expressed at a high level and SNC1 is constitutively activated [12]. Disruption of protein-protein interactions between SRFR1 and SNC1 [12] could lead to SNC1 activation; however, increased mRNA levels can also lead to SNC1 auto-activation [24,42] and based on SRFR1's interaction with TCP transcription factors a direct regulation of $S N C 1$ transcript levels $[15,43]$ is consistent with the data obtained in the eds1-2 background. Because in wild type plants levels of $S N C 1$ are kept low to avoid fitness penalties, the effects of TPR2 mutations are only apparent when SNC1 transcription is induced, such as in the autoimmune mutants srfrr-4 and snc1-1. We hypothesize that TPR2, and to some degree TPR3, acts downstream of SNC1 transcription by repressing expression of a positive regulator of $S N C 1$ or activating a negative regulator. Parsimoniously, the physical interaction of TPR2 with the TIR domain of SNC1 shown here raises the possibility that TPR2 competes with TPR1 for binding of SNC1, and that TPR1-SNC1 and TPR2-SNC1 complexes regulate target genes such as DND1 and DND2 in opposite ways.

Interestingly, in vivo data provide evidence for an alternative model in which the interaction between TPR1 and TPR2 leads to either sequestration of TPR1 to reduce formation of SNC1-TPR1 complexes or to sequestration of heteromeric SNC1-TPR1-TPR2 complexes with altered inhibitory functions. Given the context-specific degree of redundancy in the TPL family, it is worth emphasizing that the model developed here is perhaps limited to immune phenotypes or more narrowly to consequences of interactions with SNC1. Interestingly, members of the TPL-related metazoan Groucho/Transducin-Like Enhancer of split (Gro/TLE) transcriptional co-repressor family are known to be regulated by context-specific parameters, such as the levels of available partner repressors, post-translational protein modifications, or competition with activators $[44,45]$. 
In this regard, the recent demonstration of TPR1 regulation by SUMOylation is noteworthy [20]. SUMOylation reduces TPR1 repressor activity, resulting in increased expression of genes such as DND1 and DND2 and a dampened immune activation. In contrast to expectations, a version of TPR1 that cannot be SUMOylated interacts less with SNC1, suggesting that strong association of SUMOylated TPR1 with SNC1 leads to a sequestering of the co-repressor complex. A SNC1-TPR1-TPR2 heteromer may therefore either precipitate SUMOylation of TPR1 or mimic the inhibited SNC1-TPR1 co-repressor complex through structural differences between TPR1 and TPR2. It is perhaps relevant that we observed stronger in vivo interaction of TPR2 with SNC1 when TPR1 was present, although this requires more careful quantification.

Finally, enhancement of the snc1-1 phenotype by tpr2-2 illustrates that the enhanced resistance phenotype is not dependent upon mutations in SRFR1. Together, this suggests that TPR2 and SRFR1 are involved in separate pathways converging on regulation of SNC1. The mechanism of TPR2's regulation of SNC1-mediated autoimmunity merits further study.

\section{Materials and methods}

\section{Plant lines}

Plant lines used for genetic analysis were tpl-8 (SALK_036566), tpr1-2 (SALK_065650C), tpr21 (SALK_112730), tpr2-2 (SALK_079848C), tpr3-1 (SALK_029936), tpr4-1 (SALK_150008), snc1-11 (SALK_047058) from the Salk T-DNA knockout collection [46]. The srfr1-4 line (SAIL_412-E08) was from the Syngenta Arabidopsis Insertion Library [47]. Salk and SAIL lines were acquired from the Arabidopsis Biological Resource Center. The eds1-2 line was a gift from Jane Parker, and the snc1-1 line was a gift from Harrold van den Burg. All mutants are in the Col-0 background, and genotyping primers used for these lines are detailed in S1 Table. After parental lines were crossed, plants were genotyped in the F1 generation to verify the success of the cross, and then in the F2 generation to identify plants homozygous for the desired mutations. For plant growth and in planta bacterial growth assays, plants were grown in environmentally controlled conditions (Controlled Environments Ltd., Winnipeg, Manitoba, Canada; $8 \mathrm{~h}$ light / $16 \mathrm{~h}$ dark, $90-140 \mu$ mol photons $\mathrm{m}^{-2} \mathrm{~s}^{-1} ; 21^{\circ} \mathrm{C}, 70 \%$ humidity). Strain DC3000 of $P$. syringae was infiltrated into leaves of 4 week-old plants with a needle-less syringe at a bacterial density of $5 \times 10^{4}$ colony-forming units per $\mathrm{ml}$ resuspended in $10 \mathrm{mM} \mathrm{MgCl}$. Tissue samples were collected at day 0 and day 3 post inoculation and analyzed as described previously [12].

\section{Molecular cloning and generation of transgenic lines}

The TPR2-myc construct was created by amplifying the TPR2 CDS with flanking SpeI and PacI sites at the 5' and 3' ends, respectively. The binary vector pGWB20 [48] was cut with XbaI and PacI to excise the Gateway cassette, and the SpeI-TPR2-PacI fragment was ligated into the $\mathrm{XbaI}$ and PacI sites in frame with the C-terminal myc tags in pGWB20. Sequencing was used to verify the clone. Agrobacterium tumefaciens strain C58-C1 was transformed with the TPR2-myc construct by electroporation. The srfr1-4 tpr2-2 double mutant was grown at high temperatures to relieve stunting, and these plants were transformed by floral dip. Transgenic seed was selected on hygromycin B, and T3 homozygotes were selected by true breeding on selection plates. TPR2-myc protein expression was verified by western blot using c-Myc antibody sc-789 (Santa Cruz Biotechnology, Dallas, TX, USA).

The GST-TPR2 construct was created by amplifying the TPR2 CDS with flanking EcoRI and NotI sites with an additional base between the EcoRI site and the start codon. The EcoRITPR2-NotI fragment was cloned into pGEX-4T-3 (SigmaAldrich, St. Louis, MO, USA) 
digested with EcoRI and NotI. Similarly, a cDNA encoding the SNC1 TIR domain (amino acids 1-182) was amplified with flanking EcoRI and XhoI sites. The EcoRI-TIR-XhoI fragment was cloned into pET28a (EMD Millipore, Billerica, MA USA) digested with EcoRI and XhoI to create His-T7-SNC1 TIR.

\section{RNA extraction, cDNA preparation and $\mathrm{qPCR}$}

For qPCR experiments multiple plants from each genotype were ground together in liquid nitrogen to form one replicate. For each experiment two or three replicates were used per genotype. After grinding plant tissue in liquid nitrogen, total RNA was extracted using TRIZOL reagent (Thermo Fisher Scientific, Carlsbad, CA, USA). First strand cDNA synthesis was carried out using $2 \mu \mathrm{g}$ of total RNA and reverse transcription was performed using an oligo (dT) 15 primer and Moloney murine leukemia virus (MMLV) reverse transcriptase (Promega, Madison, WI, USA). qPCR was carried out using SYBR GREEN PCR Master Mix (Thermo Fisher Scientific) or Brilliant III Ultra-Fast SYBR Green qPCR Master Mix (Agilent, Santa Clara, CA, USA) on either an ABI 7500 or Agilent AriaMX qPCR system. Transcript levels were normalized using SAND gene (At2g28390) for qPCR experiments. LinRegPCR was used to determine PCR efficiency and cycle thresholds for each sample [49], and the $2^{-\Delta \Delta \mathrm{C}_{\mathrm{T}}}$ method was used to determine expression levels [50]. Primers used for qPCR are detailed in S2 Table.

\section{Protein pull-down and co-immunoprecipitation assays}

GST-TPR2, empty pGEX-4T-3, and T7-SNC1-TIR in E. coli strain BL21(DE3) were streaked to single colonies and then incubated overnight at $37^{\circ} \mathrm{C}$ in LB broth. $200 \mathrm{ml}$ of LB was inoculated with $2 \mathrm{ml}$ of overnight culture and incubated for approximately 3 hours to an optical density of $0.6-0.8$. IPTG at $500 \mu \mathrm{M}$ was added to each culture and flasks were grown overnight at $22^{\circ} \mathrm{C}$. Each culture was passed through a French press to lyse the cells. Extracts were centrifuged and $25 \mu \mathrm{l}$ of GST beads (G-Biosciences, St. Louis, MO USA) were added to $6 \mu \mathrm{l}$ supernatant of GST-TPR2 and empty pGEX-4T-3. Samples were incubated at $4^{\circ} \mathrm{C}$ for 1.5 hours with rotation. After washing 3 times with PBS, $6 \mu$ soluble protein T7-SNC1-TIR was added, and samples were incubated at $4^{\circ} \mathrm{C}$ for 1 hour. After washing 3 times with PBS protein was eluted from beads in Laemmli buffer and then used for protein blot with anti-GST and anti-T7 (EMD Millipore). For PR2 detection in S2 Fig, PR2 antibody AS207 208 (Agrisera, Vannas, Sweden) was used.

For co-immunoprecipitation (co-IP) assays, agrobacterium strain $\mathrm{C} 58 \mathrm{C} 1$ containing the corresponding constructs was infiltrated into $N$. benthamiana eds1-1 plants [30]. Infiltrated leaf areas were harvested two days post infiltration. Co-IP was performed as described by [51]. In brief, $3 \mathrm{~g}$ fresh tissue were ground into fine powder in liquid nitrogen and solubilized with 9 $\mathrm{ml}$ protein extraction buffer (100 mM Tris- $\mathrm{HCl}$ pH 7.5, $300 \mathrm{mM} \mathrm{NaCl}, 2$ mM EDTA pH 8.0, $1 \%$ Triton X-100, 10\% glycerol, and protease inhibitor). Protein extracts were centrifuged twice at 14,800 rpm for $15 \mathrm{~min}$. Supernatants were incubated with anti-HA (Sigma, E6779), anti-myc (Sigma, E6654) or anti-GFP (MBL, D153-8) beads at $4^{\circ} \mathrm{C}$ for $90 \mathrm{~min}$. After precipitation agarose beads were washed three times with a washing buffer $(50 \mathrm{mM}$ Tris- $\mathrm{HCl} \mathrm{pH} 7.5$, $150 \mathrm{mM} \mathrm{NaCl}, 1 \mathrm{mM}$ EDTA pH 8.0, 0.5\% Triton X-100, 5\% glycerol, and protease inhibitor). Beads were then boiled with $1 \times$ SDS loading buffer. Tagged proteins were detected with antiHA (Roche, 12013819001), anti-myc (Roche, 11814150001) or anti-GFP (Invitrogen, A11122) antibodies.

\section{Statistical analyses}

Student's t-test with the Bonferroni-Holm method to correct for multiple comparisons was used for all statistical analyses. Raw data underlying the analyses can be found in S1 Data for 
data represented in main figures, in S2 Data for shoot fresh weights in S1 Fig, and in S3 Data for in planta bacterial growth data in S6 and S7 Figs.

\section{Supporting information}

S1 Fig. Shoot weights of $t p l / t p r$ single mutants do not differ significantly from Col-0. Shoot weight from plants grown under short day conditions at $21^{\circ} \mathrm{C}$ for four weeks. Dots represent individual data points taken over two separate experiments. Whiskers on boxplots are drawn to the farthest data point within $1.5^{*}$ IQR of first and third quartiles. Letters denote significant differences as determined by Student's t-test $(\mathrm{P}<0.01)$ using the Bonferroni-Holm method to correct for multiple comparisons.

(PDF)

S2 Fig. PR2 expression in srfr 1-4 is affected by tpl and tpr2. Western blot of total protein extracted from srfr1-4, srfr1-4 tpl-8, srfr1-4 tpr1-2, srfr1-4 tpr2-2, srfr1-4 tpr3-1, and srfr1-4 tpr4-1. The large subunit of rubisco is shown as a loading control. (PDF)

S3 Fig. Comparison of stunting in srfr 1-4 tpl/tpr double mutants at $28^{\circ}$ and $21^{\circ} \mathrm{C}$. Growth phenotype of srfr1-4 and srfr1-4 tpl/tpr double mutants at $28^{\circ}$ (top row) and $21^{\circ} \mathrm{C}$ (bottom). (PDF)

S4 Fig. Molecular characterization of tpr 2 T-DNA insertion alleles. (A) T-DNA insertion locations for tpr2-1 (SALK_112730) in exon 13, and tpr2-2 (SALK_079848) in exon 21. Scale bar is 200 bp. (B) RT-PCR using TPR2 primers on the 3' side of the T-DNA insertions in tpr21 and tpr2-2 after 33 cycles. Actin was used as a control for quality of RNA and efficiency of reverse transcription.

(PDF)

S5 Fig. Phylogenetic tree of the Arabidopsis TOPLESS family. Phylogram showing evolutionary relationships amongst TOPLESS family members. The WD40 protein LEUNIG (LUG) is included as the outgroup. Tree was generated from full length cDNA sequences using www. phylogeny.fr.

(PDF)

S6 Fig. Slightly elevated SNC1 expression in tpr2-2 tpr3-1 plants does not lead to increased bacterial resistance. In planta bacterial growth assay with the indicated plant genotypes and DC3000 infiltrated at a bacterial density of $5 \times 10^{4}$ colony-forming units (cfu) per ml. Values represent averages from two independent experiments with triplicate samples, and error bars denote standard deviation. As determined by Student's t-test with the Bonferroni-Holm method to correct for multiple comparisons, none of the values were significantly different with $P \geq 0.2$.

(PDF)

S7 Fig. PR2 expression levels correlate with degree of bacterial resistance. In planta bacterial growth assay with the indicated plant genotypes and DC3000 infiltrated at a bacterial density of $5 \times 10^{4} \mathrm{cfu} / \mathrm{ml}$. Values represent averages from two independent experiments with triplicate samples, and error bars denote standard deviation. Letters denote statistically significant differences as determined by Student's t-test with the Bonferroni-Holm method to correct for multiple comparisons $(P<0.05)$.

(PDF) 
S1 Table. PCR primers used for genotyping mutant lines.

(PDF)

S2 Table. Primers used for $\mathrm{qPCR}$.

(PDF)

S1 Data. Raw data file for main figures.

(XLSX)

S2 Data. Raw data file for shoot weights shown in S1 Fig. (XLSX)

S3 Data. Raw data file for in planta bacterial growth assays in S6 and S7 Figs. (XLSX)

\section{Acknowledgments}

We thank Harrold van den Burg for the snc1-1 plant line, Brian Staskawicz for N. benthamiana eds1-1 seed, Gary Stacey for the pGWB20 vector, and Daniel Leuchtman and Sanzida Rahman for help with statistical analyses.

\section{Author Contributions}

Conceptualization: Christopher M. Garner, Walter Gassmann.

Formal analysis: Christopher M. Garner, Benjamin J. Spears.

Funding acquisition: Christopher M. Garner, Walter Gassmann.

Investigation: Christopher M. Garner, Benjamin J. Spears, Jianbin Su, Leland J. Cseke, Samantha N. Smith, Conner J. Rogan.

Methodology: Christopher M. Garner, Benjamin J. Spears, Jianbin Su.

Project administration: Christopher M. Garner, Walter Gassmann.

Visualization: Christopher M. Garner, Leland J. Cseke.

Writing - original draft: Christopher M. Garner.

Writing - review \& editing: Christopher M. Garner, Leland J. Cseke, Walter Gassmann.

\section{References}

1. Cui H, Tsuda K, Parker JE. Effector-triggered immunity: fom pathogen perception to robust defense. Annu Rev Plant Biol. 2015; 66:487-511. https://doi.org/10.1146/annurev-arplant-050213-040012 PMID: 25494461

2. Su J, Spears BJ, Kim SH, Gassmann W. Constant vigilance: plant functions guarded by resistance proteins. Plant J Cell Mol Biol. 2018; 93:637-650. https://doi.org/10.1111/tpj.13798 PMID: 29232015

3. Li X, Kapos P, Zhang Y. NLRs in plants. Curr Opin Immunol. 2015; 32:114-121. https://doi.org/10. 1016/j.coi.2015.01.014 PMID: 25667191

4. Huot B, Yao J, Montgomery BL, He SY. Growth-defense tradeoffs in plants: a balancing act to optimize fitness. Mol Plant. 2014; 7:1267-1287. https://doi.org/10.1093/mp/ssu049 PMID: 24777989

5. Lozano-Durán R, Zipfel C. Trade-off between growth and immunity: role of brassinosteroids. Trends Plant Sci. 2015; 20:12-19. https://doi.org/10.1016/j.tplants.2014.09.003 PMID: 25278266

6. Karasov TL, Chae E, Herman JJ, Bergelson J. Mechanisms to Mitigate the Trade-Off between Growth and Defense. Plant Cell. 2017; 29:666-680. https://doi.org/10.1105/tpc.16.00931 PMID: 28320784

7. Guo Q, Yoshida Y, Major IT, Wang K, Sugimoto K, Kapali G, et al. JAZ repressors of metabolic defense promote growth and reproductive fitness in Arabidopsis. Proc Natl Acad Sci U S A. 2018; 115:E10768E10777. https://doi.org/10.1073/pnas.1811828115 PMID: 30348775 
8. van Butselaar $T$, Van den Ackerveken $G$. Salicylic acid steers the growth-immunity tradeoff. Trends Plant Sci. 2020; 25:566-576. https://doi.org/10.1016/j.tplants.2020.02.002 PMID: 32407696

9. van Wersch R, Li X, Zhang Y. Mighty Dwarfs: Arabidopsis autoimmune mutants and their usages in genetic dissection of plant immunity. Front Plant Sci. 2016; 7:1717. https://doi.org/10.3389/fpls.2016. 01717 PMID: 27909443

10. Kwon SI, Koczan JM, Gassmann W. Two Arabidopsis srfr (suppressor of rps4-RLD) mutants exhibit avrRps4-specific disease resistance independent of RPS4. Plant J Cell Mol Biol. 2004; 40:366-375. https://doi.org/10.1111/j.1365-313X.2004.02213.x PMID: 15469494

11. Kim SH, Kwon SI, Bhattacharjee S, Gassmann W. Regulation of defense gene expression by Arabidopsis SRFR1. Plant Signal Behav. 2009; 4:149-150. https://doi.org/10.4161/psb.4.2.7682 PMID: 19649196

12. Kim SH, Gao F, Bhattacharjee S, Adiasor JA, Nam JC, Gassmann W. The Arabidopsis resistance-like gene SNC1 is activated by mutations in SRFR1 and contributes to resistance to the bacterial effector AvrRps4. PLoS Pathog. 2010; 6:e1001172. https://doi.org/10.1371/journal.ppat.1001172 PMID: 21079790

13. Li Y, Tessaro MJ, Li X, Zhang Y. Regulation of the expression of plant resistance gene SNC1 by a protein with a cnserved BAT2 domain. Plant Physiol. 2010; 153:1425-1434. https://doi.org/10.1104/pp. 110.156240 PMID: 20439546

14. Bhattacharjee S, Halane MK, Kim SH, Gassmann W. Pathogen effectors target Arabidopsis EDS1 and alter its interactions with immune regulators. Science. 2011; 334:1405-1408. https://doi.org/10.1126/ science.1211592 PMID: 22158819

15. Kim SH, Son GH, Bhattacharjee S, Kim HJ, Nam JC, Nguyen PDT, et al. The Arabidopsis immune adaptor SRFR1 interacts with TCP transcription factors that redundantly contribute to effector-triggered immunity. Plant J Cell Mol Biol. 2014; 78:978-989. https://doi.org/10.1111/tpj.12527 PMID: 24689742

16. Lee JE, Golz JF. Diverse roles of Groucho/Tup1 co-repressors in plant growth and development. Plant Signal Behav. 2012; 7:86-92. https://doi.org/10.4161/psb.7.1.18377 PMID: 22301974

17. Causier B, Ashworth M, Guo W, Davies B. The TOPLESS interactome: a framework for gene repression in Arabidopsis. Plant Physiol. 2012; 158:423-438. https://doi.org/10.1104/pp.111.186999 PMID: 22065421

18. Martin-Arevalillo R, Nanao MH, Larrieu A, Vinos-Poyo T, Mast D, Galvan-Ampudia C, et al. Structure of the Arabidopsis TOPLESS corepressor provides insight into the evolution of transcriptional repression. Proc Natl Acad Sci U S A. 2017; 114:8107-8112. https://doi.org/10.1073/pnas.1703054114 PMID: 28698367

19. Zhu Z, Xu F, Zhang Y, Cheng YT, Wiermer M, Li X, et al. Arabidopsis resistance protein SNC1 activates immune responses through association with a transcriptional corepressor. Proc Natl Acad Sci U S A. 2010; 107:13960-13965. https://doi.org/10.1073/pnas.1002828107 PMID: 20647385

20. Niu D, Lin X-L, Kong X, Qu G-P, Cai B, Lee J, et al. SIZ1-mediated SUMOylation of TPR1 suppresses plant immunity in Arabidopsis. Mol Plant. 2019; 12:215-228. https://doi.org/10.1016/j.molp.2018.12. 002 PMID: 30543996

21. Long JA, Ohno C, Smith ZR, Meyerowitz EM. TOPLESS regulates apical embryonic fate in Arabidopsis. Science. 2006; 312:1520-1523. https://doi.org/10.1126/science.1123841 PMID: 16763149

22. Wang $Y$, An $C$, Zhang $X$, Yao J, Zhang $Y$, Sun $Y$, et al. The Arabidopsis elongator complex subunit2 epigenetically regulates plant immune responses. Plant Cell. 2013; 25:762-776. https://doi.org/10.1105/ tpc.113.109116 PMID: 23435660

23. Oh E, Zhu J-Y, Ryu H, Hwang I, Wang Z-Y. TOPLESS mediates brassinosteroid-induced transcriptional repression through interaction with BZR1. Nat Commun. 2014; 5:4140. https://doi.org/10.1038/ ncomms5140 PMID: 24938363

24. $\mathrm{Yi} \mathrm{H}$, Richards EJ. A Cluster of disease resistance genes in Arabidopsis is coordinately regulated by transcriptional activation and RNA silencing. Plant Cell. 2007; 19:2929-2939. https://doi.org/10.1105/ tpc.107.051821 PMID: 17890374

25. Zhang Y, Goritschnig S, Dong X, Li X. A gain-of-function mutation in a plant disease resistance gene leads to constitutive activation of downstream signal transduction pathways in suppressor of npr1-1, constitutive 1. Plant Cell. 2003; 15:2636-2646. https://doi.org/10.1105/tpc.015842 PMID: 14576290

26. Yang S, Hua J. A haplotype-specific resistance gene regulated by BONZA/1 mediates temperaturedependent growth control in Arabidopsis. Plant Cell. 2004; 16:1060-1071. https://doi.org/10.1105/tpc 020479 PMID: 15031411

27. Kim T-H, Kunz H-H, Bhattacharjee S, Hauser F, Park J, Engineer $C$, et al. Natural variation in small molecule-induced TIR-NB-LRR signaling induces root growth arrest via EDS1- and PAD4-complexed R 
protein VICTR in Arabidopsis. Plant Cell. 2012; 24:5177-5192. https://doi.org/10.1105/tpc.112.107235 PMID: 23275581

28. Huang S, Chen X, Zhong X, Li M, Ao K, Huang J, et al. Plant TRAF proteins regulate NLR immune receptor turnover. Cell Host Microbe. 2016; 20:271. https://doi.org/10.1016/j.chom.2016.07.005 PMID: 27512906

29. Bartsch M, Gobbato E, Bednarek P, Debey S, Schultze JL, Bautor J, et al. Salicylic acid-independent ENHANCED DISEASE SUSCEPTIBILITY1 signaling in Arabidopsis immunity and cell death Is regulated by the monooxygenase FMO1 and the nudix hydrolase NUDT7. Plant Cell. 2006; 18:1038-1051. https://doi.org/10.1105/tpc.105.039982 PMID: 16531493

30. Schultink A, Qi T, Lee A, Steinbrenner AD, Staskawicz B. Roq1 mediates recognition of the Xanthomonas and Pseudomonas effector proteins XopQ and HopQ1. Plant J. 2017; 92:787-795. https://doi.org/ 10.1111/tpj.13715 PMID: 28891100

31. Dong X, Mindrinos M, Davis KR, Ausubel FM. Induction of Arabidopsis defense genes by virulent and avirulent Pseudomonas syringae strains and by a cloned avirulence gene. Plant Cell. 1991; 3:61-72. https://doi.org/10.1105/tpc.3.1.61 PMID: 1824335

32. Cordelier S, de Ruffray P, Fritig B, Kauffmann S. Biological and molecular comparison between localized and systemic acquired resistance induced in tobacco by a Phytophthora megasperma glycoprotein elicitin. Plant Mol Biol. 2003; 51:109-118. https://doi.org/10.1023/a:1020722102871 PMID: 12602895

33. Wang L, Kim J, Somers DE. Transcriptional corepressor TOPLESS complexes with pseudoresponse regulator proteins and histone deacetylases to regulate circadian transcription. Proc Natl Acad Sci. 2013; 110:761-766. https://doi.org/10.1073/pnas.1215010110 PMID: 23267111

34. Garner CM, Kim SH, Spears BJ, Gassmann W. Express yourself: Transcriptional regulation of plant innate immunity. Semin Cell Dev Biol. 2016; 56:150-162. https://doi.org/10.1016/j.semcdb.2016.05. 002 PMID: 27174437

35. Noël L, Moores TL, van Der Biezen EA, Parniske M, Daniels MJ, Parker JE, et al. Pronounced intraspecific haplotype divergence at the RPP5 complex disease resistance locus of Arabidopsis. Plant Cell. 1999; 11:2099-2112. PMID: 10559437

36. Zou B, Yang D-L, Shi Z, Dong H, Hua J. Monoubiquitination of histone $2 \mathrm{~B}$ at the disease resistance gene locus regulates its expression and impacts immune responses in Arabidopsis. Plant Physiol. 2014; 165:309-318. https://doi.org/10.1104/pp.113.227801 PMID: 24664204

37. Li X, Clarke JD, Zhang Y, Dong X. Activation of an EDS1-mediated R-gene pathway in the snc1 mutant leads to constitutive, NPR1-independent pathogen resistance. Mol Plant-Microbe Interact MPMI. 2001; 14:1131-1139. https://doi.org/10.1094/MPMI.2001.14.10.1131 PMID: 11605952

38. Parker JE, Holub EB, Frost LN, Falk A, Gunn ND, Daniels MJ. Characterization of eds1, a mutation in Arabidopsis suppressing resistance to Peronospora parasitica specified by several different RPP genes. Plant Cell. 1996; 8:2033-2046. https://doi.org/10.1105/tpc.8.11.2033 PMID: 8953768

39. Aarts N, Metz M, Holub E, Staskawicz BJ, Daniels MJ, Parker JE. Different requirements for EDS1 and $N D R 1$ by disease resistance genes define at least two $R$ gene-mediated signaling pathways in Arabidopsis. Proc Natl Acad Sci U S A. 1998; 95:10306-10311. https://doi.org/10.1073/pnas.95.17.10306 PMID: 9707643

40. Falk A, Feys BJ, Frost LN, Jones JD, Daniels MJ, Parker JE. EDS1, an essential component of $R$ genemediated disease resistance in Arabidopsis has homology to eukaryotic lipases. Proc Natl Acad Sci U S A. 1999; 96:3292-3297. https://doi.org/10.1073/pnas.96.6.3292 PMID: 10077677

41. Feys BJ, Moisan LJ, Newman MA, Parker JE. Direct interaction between the Arabidopsis disease resistance signaling proteins, EDS1 and PAD4. EMBO J. 2001; 20:5400-5411. https://doi.org/10.1093/ emboj/20.19.5400 PMID: 11574472

42. Li Y, Yang S, Yang H, Hua J. The TIR-NB-LRR gene SNC1 is regulated at the transcript level by multiple factors. Mol Plant-Microbe Interact MPMI. 2007; 20:1449-1456. https://doi.org/10.1094/MPMI-20-111449 PMID: 17977156

43. Zhang N, Wang Z, Bao Z, Yang L, Wu D, Shu X, et al. MOS1 functions closely with TCP transcription factors to modulate immunity and cell cycle in Arabidopsis. Plant J Cell Mol Biol. 2018; 93:66-78. https://doi.org/10.1111/tpj.13757 PMID: 29086441

44. Gasperowicz M, Otto F. Mammalian Groucho homologs: redundancy or specificity? J Cell Biochem. 2005; 95:670-687. https://doi.org/10.1002/jcb.20476 PMID: 15861397

45. Cinnamon E, Paroush Z. Context-dependent regulation of Groucho/TLE-mediated repression. Curr Opin Genet Dev. 2008; 18:435-440. https://doi.org/10.1016/j.gde.2008.07.010 PMID: 18721877

46. Alonso JM, Stepanova AN, Leisse TJ, Kim CJ, Chen H, Shinn P, et al. Genome-wide insertional mutagenesis of Arabidopsis thaliana. Science. 2003; 301:653-657. https://doi.org/10.1126/science.1086391 PMID: 12893945 
47. Sessions A, Burke E, Presting G, Aux G, McElver J, Patton D, et al. A high-throughput Arabidopsis reverse genetics system. Plant Cell. 2002; 14:2985-2994. https://doi.org/10.1105/tpc.004630 PMID: 12468722

48. Nakagawa T, Kurose T, Hino T, Tanaka K, Kawamukai M, Niwa Y, et al. Development of series of gateway binary vectors, $p G W B s$, for realizing efficient construction of fusion genes for plant transformation. J Biosci Bioeng. 2007; 104:34-41. https://doi.org/10.1263/jbb.104.34 PMID: 17697981

49. Ruijter JM, Ramakers C, Hoogaars WMH, Karlen Y, Bakker O, van den Hoff MJB, et al. Amplification efficiency: linking baseline and bias in the analysis of quantitative PCR data. Nucleic Acids Res. 2009; 37:e45. https://doi.org/10.1093/nar/gkp045 PMID: 19237396

50. Livak KJ, Schmittgen TD. Analysis of relative gene expression data using real-time quantitative PCR and the $2^{-\Delta \Delta \mathrm{C}_{\top}}$ Method. Methods 2001; 25:402-408. https://doi.org/10.1006/meth.2001.1262 PMID: 11846609

51. Yang M, Li C, Cai Z, Hu Y, Nolan T, Yu F, et al. SINAT E3 ligases control the light-mediated stability of the brassinosteroid-activated transcription factor BES1 in Arabidopsis. Dev Cell. 2017; 41:47-58.e4. https://doi.org/10.1016/j.devcel.2017.03.014 PMID: 28399399 